Article

\title{
Geometric Characterization of Injective Banach Lattices
}

\author{
Anatoly Kusraev ${ }^{1, *,+}$ (i) and Semën Kutateladze ${ }^{2,+}$ (iD) \\ 1 Southern Mathematical Institute and Khetagurov North-Ossetian State University, 44 Vatutina St., \\ Vladikavkaz 362025, Russia \\ 2 Sobolev Institute of Mathematics, 4 Koptyug Avenue, Novosibirsk 630090, Russia; sskut@math.nsc.ru \\ * Correspondence: kusraev@smath.ru \\ + These authors contributed equally to this work.
}

check for

updates

Citation: Kusraev, A.; Kutateladze, S. Geometric Characterization of Injective Banach Lattices. Mathematics 2021, 9, 250. https://doi.org/ $10.3390 /$ math 9030250

Received: 16 December 2020 Accepted: 15 January 2021 Published: 27 January 2021

Publisher's Note: MDPI stays neutral with regard to jurisdictional clai$\mathrm{ms}$ in published maps and institutional affiliations.

\section{Copyright: $@ 2020$ by the authors. Li-} censee MDPI, Basel, Switzerland. This article is an open access article distributed under the terms and conditions of the Creative Commons Attribution (CC BY) license (https:// creativecommons.org/licenses/by/ $4.0 /)$.

\begin{abstract}
This is a continuation of the authors' previous study of the geometric characterizations of the preduals of injective Banach lattices. We seek the properties of the unit ball of a Banach space which make the space isometric or isomorphic to an injective Banach lattice. The study bases on the Boolean valued transfer principle for injective Banach lattices. The latter states that each such lattice serves as an interpretation of an $A L$-space in an appropriate Boolean valued model of set theory. External identification of the internal Boolean valued properties of the corresponding $A L$-spaces yields a characterization of injective Banach lattices among Banach spaces and ordered Banach spaces. We also describe the structure of the dual space and present some dual characterization of injective Banach lattices.
\end{abstract}

Keywords: injective banach lattice; $L$-projection; $M$-projection; gordon's theorem; boolean valued analysis; boolean valued representation

\section{Introduction}

An injective Banach lattice is an injective object in the category of Banach lattices with positive contractions as morphisms. Arendt [1] (Theorem 2.2) proved that the injective objects are the same if we take the regular operators with contractive modulus as morphisms. The first example of an injective Banach lattice was provided by Abramovich who did not use the term in [2]: A Dedekind complete AM-space with unit is an injective Banach lattice. Later Lotz rediscovered this fact in [3] where the concept of injective Banach lattice was introduced. Lotz also proved that each AL-space is an injective Banach lattice; see [3] (Proposition 3.2). Hence, injective Banach lattices differ essentially from injective Banach spaces. Indeed, $C(K)$, the Banach lattice of real-valued continuous functions on an extremally disconnected Hausdorff compact space $K$ is the only injective object (up to isomorphism) in the category of Banach spaces and linear contractions (see the Nachbin-Goodner-Kelley-Hasumi Theorem [4] (Theorem 6)). The important contribution to the study of injective Banach lattices belongs to Cartwright [5] who found the order intersection property and proved that a Banach lattice $X$ is injective if and only if $X$ has the order intersection property and there exists a contractive positive projection to $X$ in $X^{\prime \prime}$ (the property $(P)$ ); see [6] (Definition 5.10.9(3), Theorems 5.10.10, and 5.10.11). Another significant advance is due to Haydon [7]. He discovered that an injective Banach space has a mixed $A M-A L$-structure and proved three theorems on the representation of injective Banach lattices as the space of order continuous operators between Dedekind complete Banach lattices with strong order units [7] (Theorems 5C), the space of integrable functions with respect to a Stone-algebra-valued modular measure [7] (Theorems 6H), and the space of all continuous sections of a continuous bundle of $A L$-spaces [7] (Theorems 7B).

The Boolean valued approach to the theory of injective Banach lattices was initiated in [8,9]. By the main result announced in [8] (Theorem 1) and proved in [9] (Theorem 4.4), each theorem about $A L$-spaces within Zermelo-Fraenkel set theory has its counterpart 
for injective Banach lattices. Translation of theorems from $A L$-spaces to injective Banach lattices is carried out by the machinery of Boolean valued analysis; see [6] (\$ 5.10-5.13). For other contributions of the Boolean valued approach to injective Banach lattices see $[6,10,11]$ and for other aspects of injective Banach lattices see [12-15].

The present article is a continuation of the paper [11] which deals with the geometric characterization of Banach spaces whose duals are injective Banach lattices. The article is organized as follows: Section 2 collects some Boolean valued requisites that include the Boolean valued interpretation of Banach spaces, Banach lattices, and $A L$-spaces. Section 3 yields the necessary and sufficient conditions for the unit ball in a Banach space be such that the space will admit some partial ordering that make the space an injective Banach lattice. Section 4 characterizes injective Banach lattices among ordered Banach spaces by Boolean valued interpretation of the result by Ellis that an ordered Banach space is an $A L$-space if and only if it is regular, enjoys the Riesz decomposition property, and its norm is additive on the positive cone. Section 5 contains an isomorphic characterization of injective Banach lattices by interpreting in an appropriate Boolean valued model the Timofte result that a directed, monotone complete ordered normed space $X$ with the Riesz decomposition property is order and topologically isomorphic to some $A L$-space if and only if the positive cone of $X$ is generated by some bounded closed convex subset not containing the zero. We start Section 6 with a description of the dual space, and then give a dual characterization of an injective Banach lattice.

For the theory of Banach lattices and positive operators we refer to the books [16-18]. The prerequisites of the theory of Boolean valued models are briefly presented in [19] (Chapter 9) and [6] (Chapter 1); see also [20-22] for more detail.

Throughout the sequel, $U=U(X)$ and $U^{\prime}=U\left(X^{\prime}\right)$ stand for the closed unit balls of a normed space $X$ and its topological dual $X^{\prime}$. Let $X_{+}$denote the positive cone of an ordered vector space $X$, and we will assume that $X_{+}$is closed. Also, $\mathbb{P}(X)$ stands for the Boolean algebra of band projections of a vector lattice $X$. We let $:=$ designate the assignment by definition, while $\mathbb{N}$ and $\mathbb{R}$ will symbolize the naturals and the reals.

\section{Some Boolean Valued Requisites}

The main purpose of this section is to provide notation and terminology as well as some results about Boolean valued representation we will need in what follows.

Throughout the sequel $\mathbb{B}$ is a complete Boolean algebra with unit $\mathbb{1}$ and zero $\mathbb{O}$, while $\mathbb{V}^{(\mathbb{B})}$ stands for the corresponding Boolean valued universe. There is a natural way of assigning to each formula $\phi\left(u_{1}, \ldots, u_{n}\right)$ of Zermelo-Fraenkel set theory with choice, ZFC in short, the Boolean truth value $\llbracket \phi\left(x_{1}, \ldots, x_{n}\right) \rrbracket \in \mathbb{B}$ with $x_{1}, \ldots, x_{n} \in \mathbb{V}^{(\mathbb{B})}$. We say that the statement $\phi\left(x_{1}, \ldots, x_{n}\right)$ is true within $\mathbb{V}^{(\mathbb{B})}$ and write $\mathbb{V}^{(\mathbb{B})} \models \phi\left(x_{1}, \ldots, x_{n}\right)$ if $\llbracket \phi\left(x_{1}, \ldots, x_{n}\right) \rrbracket=\mathbb{1}$. There is a smooth mathematical technique for revealing interplay between the interpretations of one and the same fact in the two models, the von Neumann universe $\mathbb{V}$ and the Boolean valued universe $\mathbb{V}^{(\mathbb{B})}$. The relevant ascending-and-descending machinery rests on the functors of canonical embedding $X \mapsto X^{\wedge}$ and ascent $X \mapsto X \uparrow$, acting from $\mathbb{V}$ into $\mathbb{V}(\mathbb{B})$, and of descent $X \mapsto X \downarrow$, acting from $\mathbb{V}^{(\mathbb{B})}$ into $\mathbb{V}$, see $[6,21]$ for details. The operations of descent, ascent, and canonical embedding can be naturally extended to mappings and relations, so that they are applicable to algebraic structures. So, there arise some functors of Boolean valued analysis whose interplay is of import in applications; see [21] (Chapter 3).

The basic properties of a Boolean valued universe are formulated in the three principles: The transfer principle tells us that all theorems of ZFC are true within $\mathbb{V}(\mathbb{B})$. The maximum principle asserts that if $\mathbb{V}^{(\mathbb{B})} \models(\exists x) \varphi(x)$, then there exists $x_{0} \in \mathbb{V}^{(\mathbb{B})}$ such that $\mathbb{V}^{(\mathbb{B})} \models \varphi\left(x_{0}\right)$. A partition of unity in $\mathbb{B}$ is a family $\left(b_{\xi}\right)_{\xi \in \Xi} \subset \mathbb{B}$ such that $\bigvee_{\xi \in \Xi} b_{\xi}=\mathbb{1}$ and $b_{\xi} \wedge b_{\eta}=\mathbb{O}$ whenever $\xi \neq \eta$. The mixing of a family $\left(x_{\xi}\right)_{\xi \in \Xi}$ in $\mathbb{V}^{(\mathbb{B})}$ by a partition of unity $\left(b_{\xi}\right)_{\xi \in \Xi}$ in $\mathbb{B}$ is an element $x \in \mathbb{V}^{(\mathbb{B})}$ satisfying $b_{\xi} \leq \llbracket x=x_{\xi} \rrbracket$ for all $\xi \in \Xi$. The mixing principle states that in $\mathbb{V}^{(\mathbb{B})}$ there exists a mixing of every family by each partition of unity. Thus, the maximum principle guarantees the existence of various Boolean valued "replicas" 
or "counterparts" of usual mathematical objects, while the mixing principle shows how these replicas may be constructed.

The following property of descents turns out rather useful in interpreting mathematical objects and statements in the Boolean valued universe $\mathbb{V}^{(\mathbb{B})}$; see [21] (2.3.8). Given $X \subset \mathbb{V}^{(\mathbb{B})}$, denote by mix $(X)$ the set of all mixings of all families in $X$ by arbitrary partitions of unity in $\mathbb{B}$. If $\mathscr{X} \in \mathbb{V}^{(\mathbb{B})}$ and $\llbracket \mathscr{X} \neq \varnothing \rrbracket=\mathbb{1}$, then for an arbitrary subset $X \subset \mathbb{V}^{(\mathbb{B})}$ with $\operatorname{mix}(X)=\mathscr{X} \downarrow$ and all formulas $\varphi$ and $\psi$ of ZFC we have

$$
\llbracket(\forall x \in \mathscr{X}) \psi(x) \rightarrow \varphi(x) \rrbracket=\bigwedge\{\llbracket \varphi(x) \rrbracket: x \in X, \llbracket \psi(x) \rrbracket=\mathbb{1}\} .
$$

Applying the transfer and maximum principles to the ZFC-theorem on the existence of the reals, find $\mathscr{R} \in \mathbb{V}^{(\mathbb{B})}$ called the reals within $\mathbb{V}^{(\mathbb{B})}$ which satisfies $\llbracket \mathscr{R}$ is the reals $=$ $\mathbb{1}$. The Gordon Theorem [23] states that the descent $\mathbf{R}:=\mathscr{R} \downarrow$ of $\mathscr{R}$ (with the descended operations and order) is a universally complete vector lattice. Moreover, there is a Boolean isomorphism $\chi$ from $\mathbb{B}$ onto $\mathbb{P}(\mathscr{R} \downarrow)$ such that

$$
\begin{aligned}
\chi(b) x=\chi(b) y & \Longleftrightarrow b \leq \llbracket x=y \rrbracket, \\
\chi(b) x \leq \chi(b) y & \Longleftrightarrow b \leq \llbracket x \leq y \rrbracket
\end{aligned}
$$

for all $x, y \in \mathscr{R}$ and $b \in \mathbb{B}$; see [21] (Theorem 5.2.2) and [6] (Theorem 2.2.4). Moreover, the universally complete vector lattice $\mathscr{R} \downarrow$ endowed with the descended multiplications is a semiprime $f$-algebra with ring unity $\mathbb{1}:=1^{\wedge}$. Moreover, for every $b \in \mathbb{B}$ the band projection $\chi(b)$ acts as the multiplication by the $\chi(b) \mathbb{1}$; see [6] (Theorem 2.3.2). For a special $\mathbb{B}$, the Gordon Theorem was first discovered by Takeuti; see [24,25].

The bounded descent $\Lambda \subset \mathbf{R}=\mathscr{R} \downarrow$ of $\mathscr{R} \downarrow$ is the order ideal in $\mathbf{R}$ generated by $1^{\wedge}$ and equipped with the order-unit norm $\|\cdot\|_{\infty}$ :

$$
\begin{gathered}
\Lambda:=\left\{x \in \mathbf{R}:(\exists C \in \mathbb{R})-C 1^{\wedge} \leq x \leq C 1^{\wedge}\right\} ; \\
\|x\|_{\infty}:=\inf \left\{C>0:-C 1^{\wedge} \leq x \leq C 1^{\wedge}\right\} \quad(x \in \Lambda) .
\end{gathered}
$$

Write $\Lambda=\Lambda(\mathbb{B})$, since $\Lambda$ is uniquely defined by $\mathbb{B}$. Clearly, $\Lambda$ is a Dedekind complete $A M$-space with unit $1^{\wedge}$. By the Kreŭns-Kakutani Representation Theorem $\Lambda \simeq C(K)$ with $K$ an extremally disconnected compact Hausdorff space, the Stone space of $\mathbb{B}$.

Let $(\mathscr{X},\|\cdot\|)$ be a Banach space within $\mathbb{V}(\mathbb{B})$ and $\mathbf{R}:=\mathscr{R} \downarrow$. Define the mapping $|\cdot|$ from $\mathscr{X} \downarrow$ to $\mathbf{R}$ as the descent $|\cdot|:=(\|\cdot\|) \downarrow$ of the norm $\|\cdot\|$. Then $\mathscr{X} \downarrow$ (with the descended operations) is a vector space, $|\cdot|$ is an $\mathbf{R}$-valued norm on $\mathscr{X} \downarrow$, and $(X,|\cdot|)$ is a Banach-Kantorovich space; see [11] (Definition 2.7). (Not to be confused with the concept of Kantorovich-Banach space or KB-space in short, which is, by definition, a Banach lattice with an order continuous Levi norm. These properties appear in the literature under different names; see Definition 7 and the footnote on page 89 in [26]). The bounded descent $\mathscr{X} \Downarrow$ of $\mathscr{X}$ is defined as the set $\mathscr{X} \Downarrow:=\{x \in \mathscr{X} \downarrow:|x| \in \Lambda\}$ equipped with the descended operations with the mixed norm, i.e., $|x|:=\||x|\|_{\infty}$ for all $x \in \mathscr{X} \Downarrow$; see [11] (Definition 2.6).

Observe that $\Lambda$ is a Dedekind complete $f$-algebra with ring and order unit $\mathbb{1}$. Moreover, both $(X,|\cdot|)$ and $(X,|\cdot|)$ admit the structure of $\Lambda$-module; see [6] (5.8.5, 5.8.7).

A projection $P$ on a Banach space $X$ is an idempotent in $\mathscr{L}(X)$, the Banach algebra of bounded linear operators on $X$, that is, $P \in \mathscr{L}(X)$ and $P \circ P=P$. When speaking of a Boolean algebra of projections in a Banach space $X$ we always mean some set $\mathscr{P}$ of commuting norm one projections on $X$ which is a Boolean algebra under the operations

$$
\pi \wedge \rho:=\pi \circ \rho=\rho \circ \pi, \quad \pi \vee \rho=\pi+\rho-\pi \circ \rho, \quad \pi^{*}=I_{X}-\pi \quad(\pi, \rho \in \mathscr{P})
$$

and in which the zero and the identity operators in $X$ serve as the top and bottom of $\mathscr{P}$.

A projection $\pi$ on a Banach space $X$ is an L-projection if $\|x\|=\|\pi x\|+\|x-\pi x\|$ for all $x \in X$, and $\pi$ is an M-projection if $\|x\|=\max \{\|\pi x\|,\|x-\pi x\|\}$ for all $x \in X$. The $L$-projections and $M$-projections different from the zero and the identity are referred to as nontrivial. The sets of all $L$-projections and $M$-projections on $X$ are denoted by $\mathbb{P}_{L}(X)$ 
and $\mathbb{P}_{M}(X)$. Furthermore, $\mathbb{P}_{L}(X)$ is a complete Boolean algebra, $\mathbb{P}_{M}(X)$ is a (generally not complete) Boolean algebra, and the mapping $\pi \mapsto \pi^{\prime}$ is a Boolean isomorphism from $\mathbb{P}_{L}(X)$ onto $\mathbb{P}_{M}\left(X^{\prime}\right)$ (see [11] (Theorem 2.10)) and from $\mathbb{P}_{M}(X)$ into $\mathbb{P}_{L}\left(X^{\prime}\right)$.

Let $\mathbb{B}$ be a Boolean algebra of norm one projections in a Banach space $X$. Take $x \in X$, a family $\left(x_{\xi}\right)$ in $X$, and a partition of the unit $\left(\pi_{\xi}\right)$ in $\mathbb{B}$. If $\pi_{\xi} x=\pi_{\xi} x_{\xi}$ for all $x \beta \in \Xi$, then we say that $x$ is a mixing of $\left(x_{\xi}\right)$ by $\left(\pi_{\xi}\right)$; cf. (2) in Section 2 A subset $C \subset X$ is $\mathbb{B}$-complete or mix-complete if, given a family $\left(x_{\xi}\right)$ in $C$ and a partition of unity $\left(b_{\xi}\right)$ in $\mathbb{B}$, we have $\operatorname{mix}_{\xi}\left(b_{\xi} x_{\xi}\right) \in C$. A Banach space $X$ is $\mathbb{B}$-complete or $\mathbb{B}$-cyclic if $\mathbb{B} \subset \mathbb{P}(X)$, the mixing of a family is unique whenever it exists, and the closed unit ball $U(X)$ of $X$ is mix-complete.

The Boolean valued representation of Banach spaces is described as follows (see [19] (Theorems 8.3.1 and 8.3.2) or [11] (Theorem 5.8.11)): Recall that a linear operator $T: X \rightarrow Y$ is $\mathbb{B}$-linear if $\pi T=T \pi$ for all $\pi \in \mathbb{B}$; here $X$ and $Y$ are Banach spaces with Boolean algebras of projections and the latter are identified with $\mathbb{B}$. A one-to-one $\mathbb{B}$-linear operator is a $\mathbb{B}$ isomorphism. An isometric $\mathbb{B}$-isomorphism of Banach spaces is a $\mathbb{B}$-isometry, and a $\mathbb{B}$-isometric lattice homomorphism between Banach lattices is a lattice $\mathbb{B}$-isometry.

(1) The bounded descent $\mathscr{X} \Downarrow$ of a Banach space $\mathscr{X}$ without nontrivial M-projections within $\mathbb{V}^{(\mathbb{B})}$ is a Banach space with the $\mathbb{B}$-complete unit ball and $\mathbb{P}_{M}(\mathscr{X} \Downarrow)$ isomorphic to $\mathbb{B}$. Conversely, if $X$ is a Banach space whose unit ball is $\mathbb{B}$-complete with $\mathbb{B}=\mathbb{P}_{M}(X)$, then there exists a Banach space $\mathscr{X}$ without nontrivial M-projections within $\mathbb{V}^{(\mathbb{B})}$ whose $M$-descent is linearly $\mathbb{B}$-isometric to $X$ and $\mathscr{X}$ is unique up to linear isometry.

As regards an alternative approach to Boolean valued interpretation of Banach space theory with very important applications to the module structure of von Neumann algebras see [27] and the references therein. We proceed with the result of [9] (Theorem 3.1) about the Boolean valued representation of Banach lattices. To this end, put $\mathbb{M}(X):=\mathbb{P}(X) \cap \mathbb{P}_{M}(X)$.

(2) The bounded descent $\mathscr{X} \Downarrow$ of a Banach lattice $\mathscr{X}$ from $\mathbb{V}^{(\mathbb{B})}$ is a $\mathbb{B}$-cyclic Banach lattice. Conversely, if $X$ is a $\mathbb{B}$-cyclic Banach lattice, then in $\mathrm{V}^{(\mathbb{B})}$ there exists a Banach lattice $\mathscr{X}$ unique up to lattice isometry whose bounded descent $\mathscr{X} \Downarrow$ is lattice $\mathbb{B}$-isometric to $X$. Moreover, $\mathbb{B} \simeq \mathbb{M}(X)$ if and only if $\llbracket$ there is no $M$-projection in $\mathscr{X}$ other than 0 and $I_{\mathscr{X}} \rrbracket=\mathbb{1}$.

Recall the conventional definition of an injective Banach lattice. A real Banach lattice $X$ is injective if, for every Banach lattice $Y$, every closed vector sublattice $Y_{0} \subset Y$, and every positive linear operator $T_{0}: Y_{0} \rightarrow X$ there is a positive linear extension $T: Y \rightarrow X$ of $T_{0}$ with $\left\|T_{0}\right\|=\|T\|$. The transfer principle for injective Banach lattices reads as follows (see [9] (Theorem 4.4) and [6] (Theorem 5.12.5)):

A bounded descent $\mathscr{X} \Downarrow$ of an $A L$-space $\mathscr{X}$ from $\mathbb{V}^{(\mathbb{B})}$ is an injective Banach lattice with $\mathbb{B} \simeq \mathbb{M}(\mathscr{X} \Downarrow)$. Conversely, if $X$ is an injective Banach lattice and $\mathbb{B} \simeq \mathbb{M}(X)$, then there is an $A L$-space $\mathscr{X}$ within $\mathbb{V}^{(\mathbb{B})}$ whose bounded descent is lattice $\mathbb{B}$-isometric to $X$.

Among the useful consequences of this fact we have the following representation result: An operator $T: X \rightarrow \Lambda$ enjoys the Levy property if $\Lambda=T(X)^{\perp \perp}$ and each increasing net $\left(x_{\alpha}\right)$ in $X$ has the supremum $\sup _{\alpha} x_{\alpha}$ provided that the net $\left(T x_{\alpha}\right)$ is order bounded in $\Lambda$. Given an injective Banach lattice $(X,\|\cdot\|)$ with $\mathbb{B}=\mathbb{M}(X)$, there is a strictly positive Maharam operator $\Phi: X \rightarrow \Lambda$ (i.e., an interval preserving order continuous linear operator such that $\Phi|x|=0$ implies $x=0$ ) enjoying the Levi property and satisfying $\|x\|=$ $\|\Phi(|x|)\|_{\infty}$ for all $x \in X$; see [9] (Corollary 4.5). In this event we say that $X$ is representable as $L^{1}(\Phi)$.

\section{Banach Space Characterization}

In this section we will present the necessary and sufficient conditions on the unit ball of a Banach space $X$ for $X$ to admit a partial ordering making $X$ into an injective Banach lattice. We start with recalling a similar characterization of $A L$-spaces.

Definition 1. A subset $C \subset X$ is $\Lambda$-convex if $\lambda x+\mu y \in C$ for all $x, y \in C$ and $\lambda, \mu \in \Lambda_{+}$with $\lambda+\mu=\mathbb{1}$. A nonempty $\Lambda$-convex subset $F$ of a $\Lambda$-convex set $C$ is a $\Lambda$-face of $C$ if whenever $z \in F$ and $z=\lambda x+\mu y$ with $x, y \in C$ and $\lambda, \mu \in \Lambda_{+}, \lambda+\mu=\mathbb{1}$, it follows that $\lambda x \in \lambda F$ and 
$\mu y \in \mu F$. In the case when $\Lambda=\mathbb{R}$, we speak of convex sets and faces. The cone $C$ is minihedral whenever the intersection of every two translates of $C$ is a translate of $C$; i.e., for all $x, y \in X$ there is $z \in X$ satisfying $(x+C) \cap(y+C)=z+C$.

We also define the $\Lambda$-convex hull $\operatorname{co}_{\Lambda}(A)$ of a set $A \subset X$ as the intersection of all $\Lambda$-convex sets in $X$ including $A$ and put $c o:=c o_{\mathbb{R}}$. Observe that if $A=c o_{\Lambda}(F \cup D)$ for some $\Lambda$-convex $F$ and $D$ then $A=\bigcup\{\alpha F+(\mathbb{1}-\alpha) D: \alpha \in \Lambda, 0 \leq \alpha \leq \mathbb{1}\}$.

Theorem 1. Let $X$ be a Banach space. Then $X$ is linearly isometric to an AL-space if and only if there is a maximal face $F \subset S$ such that $B(X)=\operatorname{co}(F \cup(-F))$ and the cone $C=\{\alpha x: \alpha \in$ $\left.\mathbb{R}_{+}, x \in F\right\}$ is minihedral.

Proof. See [4] (§ 18, Theorem 1).

Lemma 1. Let $X$ be a $\mathbb{B}$-complete Banach space and $C$ a $\mathbb{B}$-complete convex subset of $X$. Then an arbitrary maximal $\Lambda$-face of $X$ is $\mathbb{B}$-complete.

Proof. Assume that $F$ is a maximal $\Lambda$-face of $C$ and denote by $\bar{F}$ the subset of $C$ consisting of all mixtures of families in $F$ by partitions of unity in $\mathbb{B}$. It suffices to prove that $F=\bar{F}$. Consider $z \in \bar{F}$ determined by a partition of unity $\left(\pi_{\xi}\right)$ in $\mathbb{B}$ and a family $\left(z_{\xi}\right)$ in $F$; i.e., $\pi_{\xi} z=\pi_{\xi} z_{\xi}$ for all $\xi$. Suppose that $z=\alpha x+(\mathbb{1}-\alpha) y$ for some $x, y \in C$ and $\alpha \in \Lambda$ with $0 \leq \alpha \leq \mathbb{1}$. Fix $\xi$ and put $\bar{x}=\pi_{\xi} x+\bar{\pi}_{\xi} z_{\xi}$ and $\bar{y}=\pi_{\xi} y+\bar{\pi}_{\xi} z_{\xi}$ where $\bar{\pi}_{\xi}:=\mathbb{1}-\pi_{\xi}$. Then $\alpha \bar{x}+(\mathbb{1}-\alpha) \bar{y}=\pi_{\xi} z+\bar{\pi}_{\xi} z_{\xi}=z_{\xi}$ and hence $\alpha \bar{x}=\alpha z_{\xi}$ and $(\mathbb{1}-\alpha) \bar{y}=(\mathbb{1}-\alpha) \bar{y}$. It follows that $\pi_{\xi} \alpha z=\pi_{\xi} \alpha z_{\xi}=\pi_{\xi} \alpha \bar{x}=\pi_{\xi} \alpha x$, whence $\pi_{\xi}(\alpha z-\alpha x)=0$ for each fixed $\xi$, so that $\alpha z=\alpha x$ as $\left(\pi_{\xi}\right)$ is a partition of unity. Similarly, $(\mathbb{1}-\alpha) z=(\mathbb{1}-\alpha) x$. Thus $\bar{F}$ is a $\Lambda$-face of $C$; and, as $F \subset \bar{F}$ and $F$ is a maximal $\Lambda$-face of $C$, we conclude that $F=\bar{F}$ and $F$ is a $\Lambda$-face of $C$.

Let $\left(\mathscr{X},\|\cdot\|_{\mathscr{X}}\right)$ be a Banach space within $\mathbb{V}^{(\mathbb{B})}$ and let $X=\mathscr{X} \Downarrow$ be a $\mathbb{B}$-complete Banach space with the norm $\|x\|=\||x|\|_{\infty}(x \in X)$, where $|\cdot|: X \rightarrow \Lambda$ is the descent of the internal norm $\|\cdot\| \mathscr{X}$ and $\Lambda=\mathscr{R} \Downarrow$.

Lemma 2. Let $\hat{F}$ and $\hat{G}$ be convex sets in $\mathscr{X}$ and let $\hat{S}$ stand for the internal unit sphere of $\mathscr{X}$; i.e., $\llbracket \hat{S}=\left\{x \in \mathscr{X}:\|x\|_{\mathscr{X}}=1\right\} \rrbracket=\mathbb{1}$. Then

(1) $U(\mathscr{X}) \downarrow=U(X)$.

(2) $\hat{S} \downarrow=\{x \in X:\|\pi x\|=1$ for all $\pi \in \mathbb{B}\}$.

(3) $\operatorname{co}(\hat{F} \cup \hat{G}) \downarrow=\left\{\lambda f+\mu g: f \in \hat{F} \downarrow, g \in \hat{G} \downarrow ; \lambda, \mu \in \Lambda_{+}, \lambda+\mu=\mathbb{1}\right\}$.

(4) $\hat{C} \downarrow=\left\{\alpha x: \alpha \in \Lambda_{+}, x \in \hat{F} \downarrow\right\}$ if and only if $\llbracket \hat{C}=\left\{\alpha x: \alpha \in \mathscr{R}_{+}, x \in \hat{F}\right\} \rrbracket=\mathbb{1}$.

(5) $\hat{F} \downarrow$ is a maximal $\Lambda$-face of $\hat{S} \downarrow$ if and only if $\llbracket \hat{F}$ is a maximal face of $\hat{S} \rrbracket=\mathbb{1}$.

Proof. (1): It suffices to observe that $\|x\|=\||x|\|_{\infty} \leq 1$ for $x \in X$ if and only if $|x| \leq \mathbb{1}$ and if and only if $\llbracket\|x\|_{\mathscr{X}}=1 \rrbracket=\mathbb{1}$.

(2): By definition, $x \in \hat{S} \downarrow$ means that $\llbracket x \in \hat{S} \rrbracket=\mathbb{1}$. Because $|\cdot|$ is the descent of $\|\cdot\|_{\mathscr{X}}$, the latter is equivalent to the equality $|x|=\mathbb{1}$ which occurs if and only if $\|\pi x\|=1$ for all $\pi \in \mathbb{B}$.

(3): Note that $x \in \operatorname{co}(\hat{F} \cup \hat{G}) \downarrow$ if and only if

$$
(\exists f \in \hat{F})(\exists g \in \hat{G})\left(\exists \lambda, \mu \in \mathscr{R}_{+}\right) \lambda+\mu=1 \wedge x=\lambda f+\mu g
$$

within $\mathbb{V}^{(\mathbb{B})}$. By the maximum principle this is equivalent to the existence of $f \in \hat{F} \downarrow, g \in \hat{G} \downarrow$, and $\lambda, \mu \in \mathscr{R} \downarrow+$ such that $\llbracket \lambda+\mu=1 \rrbracket=\llbracket x=\lambda f+\mu g \rrbracket=\mathbb{1}$. Thus, $x \in \operatorname{co}(\hat{F} \cup \hat{G}) \downarrow$ means that $x=\lambda f+\mu g$ for some $f \in \hat{F} \downarrow, g \in \hat{G} \downarrow$, and $\lambda, \mu \in \Lambda_{+}$with $\lambda+\mu=\mathbb{1}$.

(4): A similar argument applies.

(5): The claim $\llbracket \hat{F}$ is a maximal face of $\hat{S} \rrbracket=\mathbb{1}$ means that 


$$
\begin{aligned}
\llbracket(\forall z \in \hat{F})(\forall x, y \in \hat{S})(\forall \alpha \in \mathscr{R})(0 \leq \alpha \leq 1 & \wedge z=\alpha x+(1-\alpha) y \\
& \longrightarrow \alpha z=\alpha x \wedge(1-\alpha) z=(1-\alpha) y) \rrbracket=\mathbb{1} .
\end{aligned}
$$

Calculating the Boolean truth values for the universal quantifiers by formula (1), we arrive at the equivalent statement: For all $z \in \hat{F} \Downarrow, x, y \in \hat{S} \Downarrow$, and $\alpha \in \mathscr{R} \Downarrow$ the relation $\llbracket 0 \leq \alpha \leq 1 \wedge z=\alpha x+(1-\alpha) y \rrbracket=\mathbb{1}$ implies $\llbracket \alpha z=\alpha x \wedge(1-\alpha) z=(1-\alpha) y) \rrbracket=\mathbb{1}$. Considering that $X \subset \mathscr{X} \downarrow$ and $\Lambda \subset \mathscr{R} \downarrow$ are equipped with the descended operations and $\mathbb{1}=1^{\wedge}$ by definition, we see that the latter can be rephrased as $0 \leq \alpha \leq \mathbb{1}$ and $z=\alpha x+(\mathbb{1}-\alpha)$ implies that $\alpha z=\alpha x$ and $(\mathbb{1}-\alpha) z=(\mathbb{1}-\alpha) y$.

Theorem 2. For a real Banach space $X$ with $\mathbb{B}:=\mathbb{P}_{M}(X)$ the following are equivalent:

(1) $X$ is linearly $\mathbb{B}$-isometric to an injective Banach lattice $Y$ with $\mathbb{B} \simeq \mathbb{M}(Y)$.

(2) We have

(a) $\mathbb{B}$ is a complete Boolean algebra and the unit ball of $X$ is $\mathbb{B}$-complete;

(b) the subset $S$ of the unit sphere of $X$ consisting of all $x \in X$ such that $\|\pi x\|=1$ for

all $\pi \in \mathbb{B}$ includes a maximal face $F \subset S$ for which $U_{X}=\operatorname{co}_{\Lambda}(F \cup(-F))$;

(c) $C=\left\{\alpha x: \alpha \in \Lambda_{+}, x \in F\right\}$ is a minihedral cone.

Proof. $(1) \Longrightarrow(2)$ : If 2(1) holds, then $(a)$ in 2(2) is also true and, by Theorem 1, there exists an $A L$-space $\mathscr{X}$ within $\mathbb{V}^{(\mathbb{B})}$ such that $\mathscr{X} \Downarrow$ is linearly $\mathbb{B}$-isometric to $Y$. There is no loss of generality in assuming $X=Y$. Let $\hat{S}$ stand for the unit sphere of $\mathscr{X}$. By the transfer principle, Theorem 1 is true within $\mathbb{V}^{(\mathbb{B})}$ and the maximum principle yields the existence of $\hat{F} \in \mathbb{V}^{(\mathbb{B})}$ with $\llbracket \hat{F}$ is a maximal face of $\hat{S}$ with $U(\mathscr{X})=\operatorname{co}(\hat{F} \cup(-\hat{F}))$ and the cone $\hat{C}=\left\{\alpha x: \alpha \in \mathscr{R}_{+}, x \in \hat{F}\right\}$ is minihedral $\rrbracket=\mathbb{1}$. Note that $(b)$ and $(c)$ are straightforward from Lemma 2.

$(2) \Longrightarrow(1)$ : Assume that $(a)-(c)$ are fulfilled. Then $X$ is $\mathbb{B}$-isomeric to $\mathscr{X} \Downarrow$. Put $\hat{S}:=S \uparrow, \hat{F}:=F \uparrow$, and $\hat{C}:=C \uparrow$. According to statements (1), (2), and (5) of Lemma 2, $\hat{S}$ is the unit sphere of $\mathscr{X}$ and $\hat{F}$ is a maximal face of $\hat{S}$, since $\hat{F} \downarrow=F \uparrow \downarrow=F$ by Lemma 1 . Similarly, $B(X)=c o_{\Lambda}(F \cup(-F))$ and $\hat{C}=\left\{\alpha x: \alpha \in \mathscr{R}_{+}, x \in \hat{F}\right\}$ in view of assertions (3) and (4) of Lemma 2. Moreover, $\hat{C}$ is a minihedral cone in $\mathscr{X}$ if and only if so is $\hat{C} \Downarrow$ in $X$, since $(\hat{C}+\hat{C}) \Downarrow=\hat{C} \Downarrow$ and $((x+\hat{C}) \cap(y+\hat{C})) \Downarrow=(x+\hat{C} \Downarrow) \cap(y+\hat{C} \Downarrow)$ for all $x, y \in X$. The transfer principle enables us to apply Theorem 1 within $\mathbb{V}^{(\mathbb{B})}$ and conclude that $\mathscr{X}$ is isometric to some $A L$-space $\mathscr{Y}$ within $\mathbb{V}^{(\mathbb{B})}$. It follows that $X$ is $\mathbb{B}$-isometric to $\mathscr{Y} \Downarrow$ and $\mathscr{Y} \Downarrow$ is an injective Banach lattice.

Remark 1. In Theorem 2, we can replace $U(X)=c_{\Lambda}(F \cup(-F))$ with $U(X)=\overline{c o}$ mix $x_{0}(F \cup$ $(-F))$ and define the cone $C$ as the closure of $\left\{\alpha x: \alpha \in \mathbb{R}_{+}, x \in\right.$ mix $\left._{0}(F)\right\}$, where $\overline{c o}(A)$ designates the closed convex hull of $A$, while mix $x_{0}(A)$ stands for the collection of all $\sum_{k=1}^{m} \alpha_{k} \pi_{k} a_{k}$ with $m \in \mathbb{N}, \alpha_{1}, \ldots, \alpha_{m} \in \mathbb{R}_{+}, a_{1}, \ldots, a_{m} \in A$, and $\left\{\pi_{1}, \ldots, \pi_{m}\right\}$ a finite partition of unity in $\mathbb{B}$.

\section{Ordered Banach Space Characterization}

In this section, we consider the question of when an ordered normed space is an injective Banach lattice. First, we introduce some definitions.

Definition 2. An ordered Banach space $X$ is $\mathbb{B}$-complete or $\mathbb{B}$-cyclic whenever $X$ is a $\mathbb{B}$-complete Banach space, $\mathbb{B}$ consists of positive projections, and $X_{+}$is $\mathbb{B}$-complete; see definitions in Section 3.

Lemma 3. For an ordered Banach space $X$ to be $\mathbb{B}$-complete it is necessary and sufficient that $X$ be $\mathbb{B}$-isometric and order isomorphic to the descent $\mathscr{X} \Downarrow$ of some ordered Banach space $\mathscr{X}$ within $\mathbb{V}^{(\mathbb{B})}$.

Proof. The Banach part follows from Section 2. The rest of the claim is justified in the same way as in [9] (Theorem 3.1). 
Definition 3. An ordered normed space $X$ is regular (or has a Riesz norm) if $X$ enjoys the properties:

(1) $\|\cdot\|$ is absolute monotone; i.e., if $x, y \in X$ and $\pm x \leq y$ then $\|x\| \leq\|y\|$.

(2) if $x \in X,\|x\| \leq 1$, and $0<\varepsilon \in \mathbb{R}$; then there is $y \in X$ with $\pm x \leq y$, and $\|y\|<(1+\varepsilon)$.

Lemma 4. Let $\mathscr{X}$ be an ordered Banach space within $\mathbb{V}^{(\mathbb{B})}$ and $X=\mathscr{X} \Downarrow$. The following hold:

(1) X has the Riesz decomposition property if and only if 【 $\mathscr{X}$ has the Riesz decomposition property $\rrbracket=\mathbb{1}$.

(2) $X$ is regular if and only if $\llbracket \mathscr{X}$ is regular $\rrbracket=\mathbb{1}$.

Proof. The first claim is immediate from the definitions. Regularity of $\left(\mathscr{X},\|\cdot\|_{\mathscr{X}}\right)$ means that the following formulas are true within $\mathbb{V}^{(\mathbb{B})}$ :

$$
\begin{gathered}
(\forall x, y \in \mathscr{X})-x \leq y \leq x \rightarrow\|y\|_{\mathscr{X}} \leq\|x\|_{\mathscr{X}} \\
(\forall x \in \mathscr{X})\left(\forall 0<\varepsilon \in \mathbb{R}^{\wedge}\right)\left(\|x\| \mathscr{X} \leq 1 \rightarrow(\exists y \in \mathscr{X})-x \leq y \leq x \wedge\|y\|_{\mathscr{X}}<1+\varepsilon\right) .
\end{gathered}
$$

The appearance of $\mathbb{R}^{\wedge}$ in the last formula is justified since in Definition 3(2) we can replaced $\mathbb{R}$ by a dense subset of $\mathbb{R}$. Using the descent rule (1) and given $x, y \in X$ and $c \in \mathbb{R}$, we see that $\llbracket\|x\|_{\mathscr{X}} \leq\|y\|_{\mathscr{X}} \rrbracket=\mathbb{1}$ and $\llbracket\|x\|_{\mathscr{X}} \leq c^{\wedge} \rrbracket=\mathbb{1}$ if and only if $|x| \leq|y|$ and $|x| \leq c \mathbb{1}$. Therefore, the regularity of $\mathscr{X}$ is equivalent to

$$
\begin{gathered}
(\forall x, y \in X)-x \leq y \leq x \rightarrow|y| \leq|x| \\
(\forall x \in X)(\forall 0<\varepsilon \in \mathbb{R})(|x| \leq \mathbb{1} \rightarrow(\exists y \in X)-x \leq y \leq x \wedge|y| \leq(1+\varepsilon) \mathbb{1}) .
\end{gathered}
$$

To ensure that the last two conditions are equivalent to the regularity of $(X,\|\cdot\|)$ we only have to observe that $|x| \leq c \mathbb{1}$ if and only if $\|x\| \leq c$ and $|y| \leq|x|$ if and only if $\|\pi y\| \leq$ $\|\pi x\|$ for all $\pi \in \mathbb{P}(\Lambda)$ as well as to use the equations $\|\pi x\|=\||\pi x|\|_{\infty}=\|\pi|x|\|_{\infty}$ for all $x \in X$ and $\pi \in \mathbb{B}$. The only matter that needs clarification is as follows: If condition (1) in Definition 3 is satisfied, then $\pm y \leq x$ implies $|x| \leq|y|$. Indeed, if $|x| \not|y|$, then there exist a nonzero projection $\pi \in \mathbb{B}$ and a real $\delta>0$ such that $\pi|x| \geq \pi(|y|+\delta \mathbb{1})$, whence $\|\pi x\|=\||\pi x|\|_{\infty} \geq\||\pi y|+\delta \pi \mathbb{1}\|_{\infty}=\|\pi y\|+\delta$. So we get that $\pm \pi y \leq \pi x$ while $\|\pi x\|>\|\pi y\|$, which contradicts 3(1).

Denote by $\operatorname{Prt}_{\sigma}:=\operatorname{Prt}_{\sigma}(\mathbb{B})$ and $\mathscr{P}_{\text {fin }}(X)$ the set of all countable partitions of unity in $\mathbb{B}$ and the collection of all finite subsets of $X$. Let $X$ be a $\mathbb{B}$-cyclic Banach lattice. Recall that $\|x\|=\||x|\|_{\infty}(x \in X)$, where $(X,|\cdot|)$ is a Banach-Kantorovich space with $\Lambda$-valued norm $\Lambda=\Lambda(\mathbb{B})=\mathscr{Z}_{m}(X)$. Denote

$$
\left\|x_{1}\right\| \oplus_{\mathbb{B}} \cdots \oplus_{\mathbb{B}}\left\|x_{n}\right\|:=\inf _{\left(\pi_{k}\right) \in \operatorname{Prt}_{\sigma}(\mathbb{B})} \sup _{k \in \mathbb{N}} \sum_{n=1}^{N}\left\|\pi_{k} x_{n}\right\| .
$$

In this section we will construct the injective sum of injective Banach lattices and characterize the injective Banach lattices in terms of summable sequences.

Lemma 5. Let $X$ be a $\mathbb{B}$-complete ordered Banach space. Then

(1) given a finite collection $x_{1}, \ldots, x_{N} \in X$, we have

$$
\left\|\left|x_{1}\right|+\cdots+\left|x_{N}\right|\right\|_{\infty}=\left\|x_{1}\right\| \oplus_{\mathbb{B}} \cdots \oplus_{\mathbb{B}}\left\|x_{N}\right\| ;
$$

(2) given a family $\left(x_{\alpha}\right)_{\alpha \in \mathrm{A}}$ in $X$ such that o-summable $\left(\left|x_{\alpha}\right|\right)_{\alpha \in \mathrm{A}}$ is o-summable, we have

$$
\sup _{\theta \in \mathscr{P}_{\text {fin }}(\mathrm{A})} \inf _{\left(\pi_{k}\right) \in \operatorname{Prt} \sigma} \sup _{k \in \mathbb{N}} \sum_{\alpha \in \theta}\left\|\pi_{k} x_{\alpha}\right\|=\left\|o-\sum_{\alpha \in \mathrm{A}}\left|x_{\alpha}\right|\right\|_{\infty} .
$$

Moreover, $\left(\left|x_{\alpha}\right|\right)_{\alpha \in \mathrm{A}}$ is o-summable if and only if the left hand-side in (4) is finite. 
Proof. (1): Considering finite collection $x_{1}, \ldots, x_{N} \in X$ and an arbitrary countable partition of unity $\left(\pi_{k}\right)$ in $\mathbb{B}$, we deduce

$$
\sum_{n=1}^{N}\left|x_{n}\right|=\bigvee_{k=1}^{\infty} \sum_{n=1}^{N}\left|\pi_{k} x_{n}\right|=\bigvee_{k=1}^{\infty} \pi_{k}(\mathbb{1}) \sum_{n=1}^{N}\left\|\pi_{k} x_{n}\right\|^{p} \leq \mathbb{1} \cdot \sup _{k \in \mathbb{N}} \sum_{n=1}^{N}\left\|\pi_{k} x_{n}\right\| .
$$

Hence, the inequality $\leq$ holds. To prove the reverse inequality, take an arbitrary $0<\varepsilon \in \mathbb{R}$ and choose a countable partition of unity $\left(\pi_{k}\right)$ such that

$$
\left\|\pi_{k} x_{i}\right\| \pi_{k} \mathbb{1} \leq \pi_{k}\left(\left|x_{i}\right|+\varepsilon \mathbb{1}\right) \quad(k \in \mathbb{N}, n:=1, \ldots, N) .
$$

Forming sums over $n \leq N$ and taking the supremum over $k \in \mathbb{N}$ in (5), we obtain

$$
\begin{aligned}
\sup _{k \in \mathbb{N}} \sum_{n=1}^{N}\left\|\pi_{k} x_{n}\right\| & =\left\|\bigvee_{k=1}^{\infty} \sum_{n=1}^{N}\right\| \pi_{k} x_{n}\left\|\pi_{k} \mathbb{1}\right\|_{\infty} \\
& \leq \sup _{k \in \mathbb{N}}\left\|\sum_{i=1}^{N} \pi_{k}\left(\left|x_{i}\right|+\varepsilon \mathbb{1}\right)\right\|_{\infty} \leq\left\|\sum_{i=1}^{N}\left|x_{i}\right|\right\|_{\infty}+N \varepsilon .
\end{aligned}
$$

Since $0<\varepsilon$ is arbitrary, we arrive at the required equality.

(2): According to 5(1), for every $\theta \in \mathscr{P}_{\text {fin }}$ (A) we can write

$$
\left\|\sum_{x \in \theta}|x|\right\|_{\infty}=\inf _{\left(\pi_{k}\right) \in \operatorname{Prt}_{\sigma}} \sup _{k \in \mathbb{N}} \sum_{x \in \theta}\left\|\pi_{k} x\right\| .
$$

It follows that the family $\left(\left|x_{\alpha}\right|\right)_{\alpha \in \mathrm{A}}$ is order summable if and only if the numerical family $\left(\left\|\sum_{\alpha \in \theta}\left|x_{\alpha}\right|\right\|_{\infty}\right)_{\alpha \in \mathrm{A}}$ is bounded, since $\Lambda$ is Dedekind complete. Taking the supremum in (6) over all finite subsets $\theta \subset A$ and using the fact that $\|\cdot\|_{\infty}$ is a Fatou norm we arrive at (4).

Lemma 6. Let $\left(\mathscr{X},\|\cdot\|_{\mathscr{X}}\right)$ be an ordered Banach space within $\mathbb{V}^{(\mathbb{B})}$ and let $(X,|\cdot|)$ be the restricted descent of $(\mathscr{X},\|\cdot\| \mathscr{X})$. If $\|x\|_{X}:=\||x|\|_{\infty}(x \in X)$, then for $x, y \in X$ the following are equivalent:
(1) $\|x+y\|_{\mathscr{X}}=\|x\|_{\mathscr{X}}+\|y\|_{\mathscr{X}}$ within $\mathbb{V}^{(\mathbb{B})}$;
(2) $|x+y|=|x|+|y|$.
(3) $\|x+y\|_{X}=\|x\|_{X} \oplus_{\mathbb{B}}\|y\|_{X}$.

Proof. The equivalence $(1) \Longleftrightarrow(2)$ can be easily deduced by using some simple properties of descents and ascents [6] (1.5.5(1), 1.5.6, 1.6.4, and 1.6.6), while (2) $\Longleftrightarrow$ (3) immediate from Lemma 5.

Definition 4. Let $X$ be a $\mathbb{B}$-complete normed ordered vector space. Say that the norm is $\mathbb{B}$-additive on $X_{+}$if for every $x, y \in X_{+}$we have $\|x+y\|=\|x\| \oplus_{\mathbb{B}}\|y\|$ and $X_{+}$is $\mathbb{B}$-generating if for every $u \in X$ there exist $x, y \in X_{+}$with $u=x-y$ and $\|u\|=\|x\| \oplus_{\mathbb{B}}\|y\|$.

Lemma 7. Let $X$ be a $\mathbb{B}$-complete Banach space ordered by a $\mathbb{B}$-complete positive cone $X_{+}$and let $\mathbb{B}$ consist of positive projections. Then

(1) the norm is $\mathbb{B}$-additive on $X_{+}$if and only if $|x+y|=|x|+|y|$ for all $x, y \in X_{+}$;

(2) the cone $X_{+}$is $\mathbb{B}$-generating if and only if for every $u \in X$ there exist $x, y \in X_{+}$with $u=x-y$ and $|u|=|x|+|y|$.

Proof. The claims are immediate from Lemma 6 and Definition 4.

Theorem 3. For an ordered Banach space $X$ and a complete Boolean algebra $\mathbb{B}$ the following are equivalent:

(1) $X$ is an injective Banach lattice with $\mathbb{B} \simeq \mathbb{M}(X)$.

(2) The next four conditions hold:

(a) X has the Riesz decomposition property. 
(b) $X$ is regular.

(c) $\mathbb{B} \simeq \mathbb{P}_{M}(X)$ and the unit ball $U(X)$ and the positive cone $X_{+}$are $\mathbb{B}$-complete.

(d) The norm of $X$ is $\mathbb{B}$-additive.

Proof. An ordered Banach space $X$ is $\mathbb{B}$-complete if one of the items 3(1) and 3(2a) holds. Thus, we can assume that $X=\mathscr{X} \Downarrow$ for some ordered Banach space $\mathscr{X}$ within $\mathbb{V}^{(\mathbb{B})}$. By $2.8 X$ is an injective Banach lattice if and only if $\mathscr{X}$ is $A L$-space. By the Ellis result [28] (Corollary 9.23) $\mathscr{X}$ is an $A L$-space if and only if $\mathscr{X}$ is regular and has the Riesz decomposition property while the norm $\|\cdot\|_{\mathscr{X}}$ is additive on $\mathscr{X}_{+}$. Thus, we have to show only that $\mathscr{X}$ has the above properties if and only if $X$ enjoys $3(2 b, 2 c, 2 d)$. The latter, however, follows from Lemmas 4 and 7.

Remark 2. Another way of characterizing injective Banach lattices is to use the properties of positive generation and additivity on the positive cone. An ordered Banach space $\left(X, X_{+}\right)$is an injective Banach lattice with $\mathbb{B}=\mathbb{P}_{M}(X)$ if and only if the following hold: $(a) x \wedge y$ exists in $X_{+}$for all $x, y \in X_{+} ;(b)$ the unit ball $U(X)$ and the positive cone $X_{+}$are $\mathbb{B}$-complete; $(c) X$ is $\mathbb{B}$-generated; $(d) X$ is $\mathbb{B}$-additive. This fact can be obtained as a Boolean valued interpretation of [29] (Chap 2, Theorem 6.2).

\section{Isomorphic Characterization}

In this section we give the conditions for a monotone complete normed ordered vector space to be topologically and order isomorphic to some injective Banach lattice.

Definition 5. A normed ordered vector space $X$ is monotone complete, if every monotone Cauchy sequence in $X$ is convergent. Say that $(X,|\cdot|)$ is monotone norm $\sigma 0$-complete if, given an increasing sequence $\left(x_{n}\right)_{n \in \mathbb{N}}$ in $X$ with $\left(\left|x_{n}-x_{m}\right|\right)_{(n, m) \in \mathbb{N} \times \mathbb{N}}$ order convergent to zero in $\Lambda$, there exists $x \in$ $X$ such that $\left(\left|x_{n}-x\right|\right)_{n \in \mathbb{N}}$ is order convergent to zero in $\Lambda$. In the same way we define monotone $\sigma r$-completeness, by considering relatively uniform convergence instead of order convergence.

Monotone complete ordered normed spaces arise in several different areas. Some recent advances can be found in [30].

Lemma 8. Let $\mathscr{X} \in \mathbb{V}^{(\mathbb{B})}$ be a normed ordered vector space and $X:=\mathscr{X} \Downarrow$. Then $X$ is monotone $\sigma$-complete if and only if $\llbracket \mathscr{X}$ is monotone $\sigma$-complete $\rrbracket=\mathbb{1}$.

Proof. By Lemma 3, there is a $\Lambda$-valued norm $|\cdot|$ on $X$ such that $(X,|\cdot|)$ is a BanachKantorovich space and $\|x\|=\||x|\|_{\infty}$ for all $x \in \mathscr{X} \Downarrow$; see 2.5. Using the interplay between convergent sequences in $\mathscr{R}$ and $\Lambda$ (see [19] (Propositions 8.1.4(3,4) or [6] (Propositions 2.4.5 and 2.4.6)), we can prove that $\llbracket \mathscr{X}$ is monotone complete $\rrbracket=\mathbb{1}$ if and only if $(X,|\cdot|)$ is monotone norm $\sigma o$-complete. Arguing as in [19] (Theorem 7.1.2), we can also prove that $(X,\|\cdot\|)$ is monotone complete if and only if $(X,|\cdot|)$ is norm $\sigma r$-complete. Consequently, we only have to show that the monotone $\sigma o$-completeness of $(X,|\cdot|)$ is equivalent to the monotone norm $\sigma r$-completeness of $(X,|\cdot|)$. This can be done by the same reasoning as in the proof of [19] (Theorem 2.2.3). To sketch the proof, denote by $\mathbb{B}[\mathbb{N}]$ the set of all mappings $v: \mathbb{N} \rightarrow \mathbb{B}$ such that $v(\mathbb{N})$ is a partition of unity in $\mathbb{B}$ and define the order relation on $\mathbb{B}[\mathbb{N}]$ by letting $v \leq \mu$ whenever $v(n) \wedge \mu(m) \neq 0$ implies $n \leq m$ for all $n, m \in \mathbb{N}$. Given an increasing Cauchy sequence $\left(x_{n}\right)_{n \in \mathbb{N}}$ and $v \in \mathbb{B}[\mathbb{N}]$, put $x_{v}:=\operatorname{mix} v(n) x_{n}$. Then $\left(x_{v}\right)_{v \in \mathbb{B}[\mathbb{N}]}$ is an increasing net. Let $\left(\lambda_{n}\right)$ be a decreasing sequence in $\lambda$ such that $\inf _{n} \lambda=0$ and $\left|x_{n}-x_{m}\right| \leq \lambda_{k}$ for all $n, m \geq k$. For an arbitrary $0<\varepsilon \in \mathbb{R}$ there exist a partition of unity $v_{0}:=\left(\tau_{n}\right)$ in $\mathbb{B}$ with $\tau_{n} \lambda_{n} \leq \varepsilon \tau_{n} \mathbb{1}$ for all $n \in \mathbb{N}$. Now, if $v_{1}, v_{2} \in \mathbb{B}[\mathbb{N}]$ and $v_{1}, v_{2} \geq v_{0}$, then $\left|x_{v_{1}}-x_{v_{2}}\right| \leq \varepsilon \mathbb{1}$. If $X$ is norm $r$-complete, there is $x \in X$ with $r-\lim _{v}\left|x-x_{v}\right|=0$ and, clearly, $r-\lim _{n}\left|x-x_{n}\right|=0$. 
The main result of this section will be obtained as a Boolean valued interpretation of the following two statements. Recall that $X$ is referred to as directed or generating if $X=X_{+}-X_{+}$.

Lemma 9. Assume that an normed ordered vector space $X$ is directed, monotone complete and has the Riesz decomposition property. Then $X$ is order isomorphic to some AL-space if and only if the positive cone can be written as $X_{+}=\mathbb{R}_{+} B$ for some closed bounded convex set $B \subset X_{+} \backslash\{0\}$.

Proof. This is a special case of Timofte's isomorphic characterization of $A L$-spaces among Hausdorff topological ordered vector space; see [31] (Theorem 14).

Lemma 10. If $X$ and $B$ are the same as in Lemma 9, then there is an equivalent $A L-n o r m\|\cdot\|_{L}$ on $X$ such that the following hold:

(1) $r \leq\|x\|_{L}$ for all $x \in X_{+}$and $r \in \mathbb{R}_{+}$with $x \in r B$.

(2) If there exists $1<\gamma \in \mathbb{R}$ such that $\gamma^{-1} U \subset X \backslash B$ and $\operatorname{co}(B \cup(-B)) \subset \gamma U$ then $\|x\|_{L} \leq \alpha \gamma$ for all $x \in X$ and $\alpha \in \mathbb{R}_{+}$with $x \in \alpha \operatorname{co}(B \cup(-B))$.

Proof. By hypotheses there is an equivalent $A L$-norm $\|\cdot\|_{L}$ on $X$ such that $q(x) \leq\|x\|_{L}$ for all $x \in X_{+}$, where $q(x):=\sup \left\{\alpha \in \mathbb{R}_{+}: x \in \alpha B\right\}$; see [31] (Lemma 2 and Theorem 9). This yields 10(1). As in the proof of [31] (Lemma 2), we can verify that $\|x\| \leq \gamma p(x)$ for all $x \in X$, where $p(\cdot)$ is the Minkowski functional of $\operatorname{co}(B \cup(-B))$. Now, 10(2) is immediate from the definition of the Minkowski functional.

Lemma 11. Let $\mathscr{X}$ be a Banach space within $\mathbb{V}^{(\mathbb{B})}$ and $X=\mathscr{X} \Downarrow$. If $B \subset X$ is $\mathbb{B}$-complete and $\hat{B}:=B \uparrow$, then $\llbracket \hat{B} \subset \mathscr{X}$ is closed and convex $\rrbracket=\mathbb{1}$ if and only if $B$ is closed and convex. If $B$ is bounded then $\llbracket \hat{B}$ is bounded $\rrbracket=\mathbb{1}$.

Proof. Observe that $\llbracket x \in \mathscr{X}$ is a limit point of $\hat{B} \rrbracket=\mathbb{1}$ if and only if for every $0<\varepsilon \in \mathbb{R}$ there exist a partition of unity $\left(\pi_{\xi}\right)$ in $\mathbb{B}$ and a family $\left(x_{\xi}\right)$ in $B$ such that $\left\|x-x_{\varepsilon}\right\|<\varepsilon$ and $x_{\varepsilon}=\operatorname{mix}_{\xi} \pi_{\xi} x_{\xi}$, that is, $x$ is a limit point of $\operatorname{mix}(B)=B$. Together with Lemma 1, this yields the first claim. The second claim is obvious.

Positive operators are often automatically continuous: Every positive operator from a Banach lattice to a normed vector lattice is continuous; see [16] (Corollary 4.4) and [18] (Proposition 1.3.5)). Below we need a stronger version of this fact.

Lemma 12. Let $X$ and $Y$ be two ordered Banach spaces whose positive cones are closed. If $X$ is directed then every positive operator from $X$ to $Y$ is continuous.

Proof. See [26] (Corollary 6), where the authorship of the statement is attributed to Lozanovsky.

Theorem 4. Let $\mathbb{B}$ be a complete Boolean algebra and $\Lambda=\Lambda(\mathbb{B})$. Assume that a $\mathbb{B}$-complete normed ordered vector space $X$ is regular and monotone complete. Also, $X$ has the Riesz decomposition property. Then the following are equivalent:

(1) There exists an equivalent norm $\|\cdot\|_{L}$ on $X$ such that $\left(X,\|\cdot\|_{L}\right)$ is an injective Banach lattice with $\mathbb{B}=\mathbb{P}_{M}\left(X,\|\cdot\|_{L}\right)$.

(2) There exists a bounded, closed, and convex $\mathbb{B}$-complete set $B \subset X_{+}$such that $0 \notin B$ and $X_{+}=\Lambda_{+} B:=\left\{\lambda b: \lambda \in \Lambda_{+}, b \in B\right\}$.

Proof. $(1) \Longrightarrow(2)$ : Assume $4(1)$. Then $\left(X,\|\cdot\|_{L}\right)$ is representable as $L^{1}(\Phi)$ with a strictly positive order continuous operator $\Phi: X \rightarrow \Lambda$ having the Levy property. If $B:=\{x \in$ $\left.X_{+}: \Phi(x)=\mathbb{1}\right\}$ then evidently $B$ is convex. Moreover, $B$ is closed and bounded, since the formula $\|x\|=\|\Phi(|x|)\|_{\infty}(x \in X)$ implies that $\Phi$ is continuous and $\|b\|=1$ for all $b \in B$. If $\pi x=0$ for some $\pi \in \mathbb{B}$ and $x \in B$ then $0=\Phi(\pi x)=\pi \Phi(x)=\pi(\mathbb{1})$ so that $\pi=0$. Given 
$x \in X_{+}$, a partition of unity $\left(\pi_{\xi}\right)$ in $\mathbb{B}$ and a family $\left(x_{\xi}\right)$ such that $\pi_{\xi} x=\pi_{\xi} x_{\xi}$ for all $\xi$, we have $\pi_{\xi} \Phi(x)=\Phi\left(\pi_{\xi} x\right)=\Phi\left(\pi_{\xi} x_{\xi}\right)=\pi_{\xi} \mathbb{1} \Phi(x)$ and hence $\Phi(x)=\mathbb{1}$. Finally, check that each $x \in X_{+}$can be written as $x=\lambda b$ with $\lambda \in \Lambda_{+}$and $b \in B$. Assume first that $\lambda=\Phi(x)$ is an order unit in $\Lambda$. Then there exists $\mu \in \Lambda^{u}$ such that $\lambda \mu=\mathbb{1}$ and we can pick a disjoint sequence $\left(\mu_{n}\right)$ in $\Lambda_{+}$with $\mu=\bigvee_{n} \mu_{n}$. Since $\Phi\left(\bigvee_{k=1}^{n} \mu_{k} x\right)=\sum_{k=1}^{n} \mu_{k} \Phi(x) \leq \lambda \mu=\mathbb{1}$ and $\Phi$ has the Levy property, $\bar{x}=\bigvee_{n} \mu_{k} x$ exists in $X_{+}$and $\Phi(\bar{x})=\bigvee_{n} \mu_{k} \Phi(x)=\mathbb{1}$. Thus, $\bar{x} \in B$ and $\lambda \bar{x}=\bigvee_{n} \lambda \mu_{n}=x$. To settle the general case, denote by $\pi$ the band projection onto the band generated by $\lambda$, pick $x_{0} \in X_{+}$with $\Phi\left(x_{0}\right)=\mathbb{1}$, and put $y:=x+\pi^{\prime} x_{0}, \pi^{\prime}:=I_{\Lambda}-\pi$. Then $\Phi(y)=\lambda+\pi^{\prime} \mathbb{1}$ is an order unit in $\Lambda$ and, by the above, $y=\left(\lambda+\pi^{\prime} \mathbb{1}\right) b$ for some $b \in B$. It follows that $x=\pi y=\pi \lambda b \in \Lambda_{+} B$ as desired.

$(2) \Longrightarrow(1)$ : Let $(\mathscr{X},\|\cdot\| \mathscr{X}) \in \mathbb{V}^{(\mathbb{B})}$ be a Boolean valued representation of $X$. Identify $(X,\|\cdot\|)$ with $\left(\mathscr{X},\|\cdot\|_{\mathscr{X}}\right) \Downarrow$ and put $\mathscr{X}_{+}:=X_{+} \uparrow$. Arguing as in the proof of [9] (Theorem $3.1)$, we can show that $\left(\mathscr{X}, \mathscr{X}_{+}\right)$is an normed ordered vector space within $\mathbb{V}^{(\mathbb{B})}$, and $X_{+}=\mathscr{X}_{+} \Downarrow$ as $X_{+}$is $\mathbb{B}$-complete. Moreover, $\mathscr{X}$ is regular and has the Riesz decomposition property by Lemma 4 and $\mathscr{X}$ is monotone complete by Lemma 8 . Assume $4(2)$ and put $\mathscr{B}:=B \uparrow$. Then $\mathscr{B}$ is bounded, closed, and convex by Lemma 11. If $\pi=\llbracket 0 \in \mathscr{B} \rrbracket=\mathbb{1}$ then $0 \in \pi B$ so that $\pi=0$ and $\llbracket 0 \notin \mathscr{B} \rrbracket=\mathbb{1}$. To see that $\llbracket \mathscr{X}_{+}=\mathscr{R}_{+} \mathscr{B} \rrbracket=\mathbb{1}$, we need only to check that $\llbracket \exists \lambda \in \mathscr{R}_{+} \rrbracket=\llbracket \exists b \in \mathscr{B} \rrbracket=\llbracket x=\lambda b \rrbracket=\mathbb{1}$ for all $x \in X_{+}$or, which is the same, $X_{+} \subset \mathscr{R} \downarrow_{+} B$. But even more is true: $X_{+}=\Lambda_{+} B$ by hypothesis. Thus, $\mathscr{X}$ meets the requirements of Lemma 9. By the transfer principle, Lemma 9 is true within $\mathbb{V}^{(\mathbb{B})}$ and so $\llbracket$ there is an equivalent $A L$-norm $\|\cdot\|_{\mathscr{L}}$ on $\mathscr{X} \rrbracket=\mathbb{1}$. In particular, $\llbracket \mathscr{X}$ is Dedekind complete vector lattice $\rrbracket=\mathbb{1}$ by $[31]$ (Proposition 8 ). Let $|\cdot|$ and $|\cdot|_{L}$ stand for the descents of $\|\cdot\|_{\mathscr{X}}$ and $\|\cdot\|_{\mathscr{L}}$.

Prove that $X=X_{L}:=\left\{x \in \mathscr{X} \downarrow:|x|_{L} \in \Lambda\right\}$. Interpreting Lemma 10(1) in $\mathbb{V}^{(\mathbb{B})}$ yields that $\lambda \leq|x|_{L}$ for all $0 \leq x \in \mathscr{X} \downarrow$ and $\lambda \in \Lambda_{+}^{u}$ with $x \in \lambda B$. For an arbitrary $x \in \mathscr{X} \downarrow$ there is a countable partition of unity $\left(\pi_{n}\right)$ in $\mathbb{B}$ such that $\pi_{n}|x| \in \Lambda$ for all $n$. Then $\pi_{n}|x| \in X_{+}$and, by hypothesis, $\pi_{n}|x|=\lambda_{n} b_{n}$ for some $b_{n} \in B$ and $\lambda_{n} \in \Lambda$. Put $\lambda=o-\sum \lambda_{n}$ and $b=o-\sum b_{n}$. Observe that $b \in B$, while $\lambda$ exists in $\Lambda^{u}$. If $|x|_{L} \in \Lambda$, then $\lambda \in \Lambda$ as $\pi_{n}|x| \in \lambda_{n} B$ and $\lambda_{n} \leq \pi_{n}|x|_{L} \leq|x|_{L} \in \Lambda$. Moreover, $\lambda b=|x|$. Thus, $\lambda \leq|x|_{L}$ so that $|x|_{L} \in \Lambda$ implies $x \in X$, i.e., $X_{L} \subset X$.

To demonstrate the converse inclusion note that we can pick $0<\gamma \in \mathbb{R}$ such that $\left(\gamma^{-1} U\right) \cap B=\varnothing$ and $B \subset \gamma U$ as $B$ is closed and convex and $0 \notin B$. Note also that $\mathrm{co}_{\Lambda}(B \cup(-B))$ consists of all elements of the form $v_{1} a_{1}+v_{2}\left(-a_{1}\right)$ with $v_{1}, v_{2} \in \Lambda_{+}, v_{1}+$ $v_{2}=\mathbb{1}$, and $a_{1}, a_{2} \in B$. Hence, $c o_{\Lambda}(B \cup(-B)) \subset \gamma U$. It follows that within $\mathbb{V}^{(\mathbb{B})}$ we have $\left(1 / \gamma^{\wedge} \mathscr{U}\right) \cap \mathscr{B}=\varnothing$ and $\operatorname{co}(\mathscr{B} \cup(-\mathscr{B})) \subset \gamma^{\wedge} \mathscr{U}$, where $\mathscr{U}$ stands for the unit ball of $\mathscr{X}$. By the transfer principle, Lemma 10 is applicable within $\mathbb{V}^{(\mathbb{B})}$ so that $\|x\|_{\mathscr{L}} \leq \alpha \gamma^{\wedge}$ for all $x \in \mathscr{X}$ and $\alpha \in \mathscr{R}_{+}$with $x \in \alpha \operatorname{co}(\mathscr{B} \cup(-\mathscr{B}))$. Descending, we get that $|x|_{L} \leq \alpha \gamma$ for all $x \in X$ and $\alpha \in \Lambda_{+}$with $x \in \alpha \operatorname{co}(B \cup(-B))$. By hypothesis, for an arbitrary $x \in X$ there are $\lambda_{1}, \lambda_{2} \in \Lambda_{+}$and $b_{1}, b_{2} \in B$ such that $x^{+}=\lambda_{1} b_{1}$ and $x^{-}=\lambda_{2} b_{2}$. Observe that $v:=\lambda_{1}+\lambda_{2}+\varepsilon \mathbb{1}$ is invertible in $\Lambda$ and we deduce

$$
x=\lambda_{1} b_{1}-\lambda_{2} b_{2}=v\left(\frac{\lambda_{1}}{v} b_{1}+\frac{\lambda_{2}}{v} b_{2}+\frac{\varepsilon \mathbb{1}}{v} 0\right) \in v c o_{\Lambda}(B \cup(-B)) .
$$

Thus, $|x|_{L} \leq \gamma v \in \Lambda$ and the inclusion $X \subset X_{L}$ follows. To complete the proof, we need only note that $\left(X,\|\cdot\|_{L}\right)$ is a Banach lattice with $\|x\|_{L}:=\left\||x|_{L}\right\|_{\infty}(x \in X)$ (see 2.8) and apply Lemma 12.

A rather different isomorphic characterization of $A L$-spaces is given by the following result due to Schlotterbeck: A Banach lattice $X$ is lattice isomorphic to an $A L$-space if and only if every positive summable sequence in $X$ is absolutely summable; see [32] (Theorem 2.7).

Definition 6. Put $\Theta:=\mathscr{P}_{\text {fin }}(\mathbb{N})$. A sequence $\left(x_{n}\right)$ in $X$ is $\mathbb{B}$-summable if there is $x \in X$ such that for every $0<\varepsilon \in \mathbb{R}$ there exists a partition of unity $\left(\pi_{\theta}\right)_{\theta \in \Theta}$ in $\mathbb{B}$ with $\left\|\pi_{\theta}\left(x-\sum_{x \in \bar{\theta}} x\right)\right\| \leq \varepsilon$ for all $\theta \in \Theta:=\mathscr{P}_{\text {fin }}(\mathbb{N})$ and $\theta \subset \bar{\theta}$. In this event $x$ is called the $\mathbb{B}$-sum of $\left(x_{n}\right)$. If the sequence $\left(x_{n}\right)$ 
is positive and the norm in $X$ is absolute monotone, then we see that that for every $0<\varepsilon \in \mathbb{R}$ there exists a countable partition of unity $\left(\pi_{n}\right)$ in $\mathbb{B}$ with $\left\|\pi_{n}\left(x-\sum_{k=1}^{N} x_{k}\right)\right\| \leq \varepsilon$ for all $n, N \in \mathbb{N}$ with $N \geq n$. A sequence $\left(x_{n}\right)$ is absolutely $\mathbb{B}$-summable if

$$
\sup _{N \in \mathbb{N}} \inf _{\left(\pi_{k}\right) \in \operatorname{Prt}_{\sigma}(\mathbb{B})} \sup _{k \in \mathbb{N}} \sum_{n=1}^{N}\left\|\pi_{k} x_{n}\right\|<+\infty .
$$

Taking $\mathbb{B}$ to be the two-element Boolean algebra $\{\mathbb{O}, \mathbb{1}\}$ in Definition 6 , we arrive at the definition of a summable and an absolute summable sequence; see [32] (page 240).

Theorem 5. For a $\mathbb{B}$-cyclic Banach lattice $X$ the following are equivalent:

(1) There is a countable partition of unity $\left(\pi_{n}\right)_{n \in \mathbb{N}}$ in $\mathbb{B}$ such that $\pi_{n} X$ is lattice $\pi_{n} \mathbb{B}$ isomorphic to an injective Banach lattice for every $n \in \mathbb{N}$.

(2) Every positive $\mathbb{B}$-summable sequence in $X$ is absolutely $\mathbb{B}$-summable.

Proof. Let $X$ be a $\mathbb{B}$-cyclic Banach lattice and let $\left(\mathscr{X},\|\cdot\|_{\mathscr{X}}\right)$ be a Banach lattice within $\mathbb{V}^{(\mathbb{B})}$, the Boolean valued representation of $X$; see 2.7(1). By Lemma $5(2)$ a sequence $\left(x_{n}\right)$ in $X$ is absolutely $\mathbb{B}$-summable if and only if $o-\sum_{n \in \mathbb{N}}\left|x_{n}\right|$ exists in $\Lambda$. But the latter is means that $\llbracket$ the sequence $\left(x_{n}\right)$ is absolutely summable in $\mathscr{X} \rrbracket=\mathbb{1}$. It is easy that $\mathbb{B}$-sum of $\left(x_{n}\right)$ exists and equals $x$ if and only if $o-\lim _{n \rightarrow \infty}\left|x-s_{n}\right|=0$ with $s_{n}:=\sum_{k=1}^{n} x_{k}$ if and only if

$$
\llbracket \lim _{n \rightarrow \infty}\left\|x-\sum_{k=1}^{n} x_{k}\right\|_{\mathscr{X}}=0 \rrbracket=\mathbb{1} .
$$

Consequently, 5(2) means that [every positive summable sequence in $\mathscr{X}$ is absolutely summable $\rrbracket=\mathbb{1}$. The transfer principle enables us to apply within $\mathbb{V}^{(\mathbb{B})}$ the Schlotterbeck characterization of $A L$-spaces, so that by the maximum principle there exists $\mathscr{L}, v \in \mathbb{V}^{(\mathbb{B})}$ such that $\llbracket \mathscr{L}$ is an $A L$-space $\rrbracket=\mathbb{1}$ and $\llbracket v: \mathscr{X} \rightarrow \mathscr{L}$ is a lattice isomorphism of $\mathscr{X}$ onto $\mathscr{L} \rrbracket=\mathbb{1}$. Assume, moreover, that $\llbracket\|v\|,\left\|v^{-1}\right\| \leq n^{\wedge} \rrbracket=\mathbb{1}$ for some $n \in \mathbb{N}$. Then $V:=v \Downarrow$ is a lattice $\mathbb{B}$-isomorphism of $\mathscr{X} \Downarrow$ onto $L:=\mathscr{L} \Downarrow$ and $\|V\|,\left\|V^{-1}\right\| \leq n$. To settle the general case, observe that the sentence $(\exists n \in \mathbb{N})\|V\|,\left\|V^{-1}\right\| \leq n$ is true, so that by the transfer principle

$$
\mathbb{1}=\llbracket\left(\exists n \in \mathbb{N}^{\wedge}\right)\|v\|,\left\|v^{-1}\right\| \leq n \rrbracket=\bigvee_{n \in \mathbb{N}} \llbracket\|v\|,\left\|v^{-1}\right\| \leq n^{\wedge} \rrbracket
$$

It follows that there is a partition of unity $\pi_{n}$ in $\mathbb{B}$ such that $\pi_{n} \leq \llbracket\|v\|,\left\|v^{-1}\right\| \leq n^{\wedge} \rrbracket$. Put $\mathbb{B}_{n}:=\pi_{n} \mathbb{B}:=\left[\mathbb{O}, \pi_{n}\right], \mathscr{X}_{n}:=\pi_{n} \mathscr{X}, \mathscr{L}_{n}:=\pi_{n} \mathscr{L}$, and $v_{n}:=\pi_{n} v$. Note that $\mathbb{V}^{\left(\mathbb{B}_{n}\right)} \models "{ }^{\prime} v_{n}$ is a lattice isomorphism from $\mathscr{X}_{n}$ onto $\mathscr{L}_{n}^{\prime \prime}$. It follows that $V_{n}:=v_{n} \Downarrow$ is a lattice $\mathbb{B}_{n^{-}}$ isomorphism from $\mathscr{X}_{n} \Downarrow$ onto $L_{n}:=\mathscr{L}_{n} \Downarrow$ and $\left\|V_{n}\right\|,\left\|V_{n}^{-1}\right\| \leq n$.

Remark 3. Condition 5(1) admits the equivalent formulation: There are a Banach lattice $\left(X_{0}, \|\right.$. $\left.\|_{0}\right)$ and a countable partition of unity $\left(\pi_{n}\right)$ in $\mathbb{B}$ such that $(a) X_{0}$ is an order dense ideal of $X$; (b) $\left(X_{0},\|\cdot\|_{0}\right)$ is an injective Banach lattice; (c) $\pi_{n} X_{0}=\pi_{n} X$ for all $n \in \mathbb{N}$; and (d) $\pi_{n} X_{0}$ and $\pi_{n} X$ are lattice $\pi_{n} \mathbb{B}$-isometric under the induced norms. To demonstrate this, it suffices to put $X_{0}:=\left\{x \in X: \sup _{n}\left\|\pi_{n} x\right\|_{L_{n}}<\infty\right\}$.

\section{Dual Characterization}

In this section, we will provide some dual characterization of injective Banach lattices. To start with, we reveal the structure of the dual space. Below, $X^{\prime}$ and $\langle\cdot, \cdot\rangle$ stand for the topological dual of $X$ and the natural duality pairing between $X$ and $X^{\prime}$.

Definition 7. The $\mathbb{B}$-dual $\left(X^{\#},|\cdot|\right)$ of a lattice normed vector space $X$ over $\Lambda$ is defined as the lattice normed vector space over $\Lambda$, where $X^{\#}$ consists of all linear operators $x^{\#}$ from $X$ to $\Lambda$ such that there exists $\lambda \in \Lambda_{+}$with $\left|\left\langle\left\langle x, x^{\#}\right\rangle\right\rangle\right|:=\left|x^{\#}(x)\right| \leq \lambda|x|$ for all $x \in X$. The least $\lambda$ satisfying the above is denoted by $\left|x^{\#}\right|$. The mixed norm on $X$ is determined, as usual, by ||$x^{\#}|\|:=\|| x^{\#} \mid \|_{\infty}$. 
Definition 8. $A \mathbb{B}$-complete $A M$-space will be referred to as $\mathbb{B}$ - $A M$-space. An $M$-norm is a $\Lambda(\mathbb{B})$ valued norm $|\cdot|$ on $X$ satisfying $|x \vee y|=|x| \vee|y|$ for all $x, y \in X_{+}$.

Theorem 6. Let $X$ be a $\mathbb{B}$-complete ordered Banach space, let $\mathscr{X} \in \mathbb{V}^{(\mathbb{B})}$ be the Boolean valued representation of $X$, and $\Lambda=\Lambda(\mathbb{B})$. Then the following are equivalent:

(1) $X$ is an injective Banach lattice with $\mathbb{P}_{M}(X)$ isomorphic to $\mathbb{B}$.

(2) $\mathscr{X}^{\prime}$ is an AM-space within $\mathbb{V}^{(\mathbb{B})}$.

(3) $\left(X^{\#},|\cdot|\right)$ is a Banach-Kantorovich lattice with a $\Lambda$-valued $M$-norm.

(4) $\left(X^{\#},\|\cdot \mid\|\right)$ is a $\mathbb{B}-A M$-space.

Proof. By Section $2, X$ is an injective Banach lattice with $\mathbb{P}_{M}(X) \simeq \mathbb{B}$ if and only if $\llbracket \mathscr{X}$ is an $A L$-space $\rrbracket=\mathbb{1}$. By the duality between $A L$ - and $A M$-spaces which was discovered by Ellis [29] (Theorem 6.4) and the transfer principle, $X$ is an injective Banach lattice with $\mathbb{P}_{M}(X) \simeq \mathbb{B}$ if and only if $\llbracket \mathscr{X}^{\prime}$ is an $A M$-space $\rrbracket=\mathbb{1}$. Hence, $(1) \Longleftrightarrow(2)$. By Section 2 and [11] (Lemma 3.5), $X^{\#}$ is a Banach-Kantorovich lattice if and only if $\llbracket \mathscr{X}^{\prime}$ is a Banach lattice $\rrbracket=\mathbb{1}$. Moreover, it is immediate from the properties of descents that $\llbracket\|\cdot\|$ is an $A M$-norm $\rrbracket=\mathbb{1}$ if and only if $|\cdot|$ is an $\Lambda$-valued $M$-norm, whence (2) $\Longleftrightarrow$ (3). Since a decomposable lattice normed vector space is order complete if and only if it is disjointly complete and relatively uniformly complete [19] (Theorem 2.3.2). Also, a vector space with a mixed norm is a Banach space if and only if the corresponding lattice normed vector space is complete under the norm $\sigma r$-convergence [19] (7.1.2). It follows that $\left(X^{\#},|\cdot|\right)$ is a Banach-Kantorovich lattice if and only if $\left(X^{\#},\|\cdot\| \mid \|\right)$ is a Banach lattice. To demonstrate the equivalence (3) $\Longleftrightarrow(4)$, it suffices to show that $|\cdot|$ and $|\|\cdot|\||$ are or are not $M$-norms simultaneously. Assume that $\|\cdot|\||$ ia an $M$-norm, but $|x| \vee|y|<|x \vee y|$ for some $x, y \in X$. Then there are $\pi \in \mathbb{B}$ and $0<\varepsilon \in \mathbb{R}$ such that

$$
|\pi x| \vee|\pi y|+\varepsilon \pi \mathbb{1}=\pi(|x| \vee|y|+\varepsilon \mathbb{1}) \leq \pi|x \vee y|=|\pi(x \vee y)| .
$$

Taking $\|\cdot\|_{\infty}$-norms, we obtain

$$
\||\pi x|\| \vee\|\pi y\|\left|+\varepsilon=\||\pi x| \vee|\pi y|+\varepsilon \pi \mathbb{1}\|_{\infty} \leq\||\pi(x \vee y)|\|_{\infty}=\|\pi(x) \vee \pi(y)\| \| .\right.
$$

This is a contradiction. The converse is obvious.

Lemma 13. Let $X$ and $Y$ be Dedekind complete vector lattices and let $\Phi$ be a Maharam operator from $X$ to $Y$. Then for every regular operator $T: X \rightarrow Y$ with $|T| \leq \Phi$ there exists an orthomorphism $w \in \mathscr{Z}(X)$ such that $|w| \leq I_{X}$ and $T x=\Phi(w x)$ for all $x \in X$.

Proof. This is a special case of the Radon-Nikodým Theorem for regular operators due to Luxemburg and Schep; see [33].

Lemma 14. Let $X, Y$, and $\Phi$ be the same as in Lemma 2. Then there exists an $f$-algebra isomorphism $h$ from $\mathscr{Z}(Y)$ onto the order complete $f$-subalgebra of $\mathscr{Z}(X)$ such that $\pi \Phi(x)=\Phi(h(\pi) x)$ holds for all $\pi \in \mathscr{Z}(Y)$ and $x \in X$.

Proof. This is an obvious consequence of [19] (Theorem 3.4.10).

Lemma 15. If $(X,\|\cdot\|)$ is a Banach space associated with a Banach-Kantorovich space $(X,|\cdot|)$ over $\Lambda$, then $X^{\prime}$ is associated with the Banach-Kantorovich space $\left(X^{\prime},|\cdot|\right)$, where the $\Lambda$-valued norm $\left|x^{\prime}\right| \in \Lambda^{\prime}$ of $x^{\prime} \in X^{\prime}$ is defined as

$$
\left\langle\lambda,\left|x^{\prime}\right|\right\rangle=\sup \left\{\left\langle x, x^{\prime}\right\rangle: x \in X,|x| \leq \lambda\right\} \quad(\lambda \in \Lambda) .
$$

In particular, $\left\|x^{\prime}\right\|=\left\|\left|x^{\prime}\right|\right\|_{\infty}$ and $\left\langle x, x^{\prime}\right\rangle \leqslant\left\langle|x|,\left|x^{\prime}\right|\right\rangle$ for all $x \in X$ and $x^{\prime} \in X^{\prime}$. 
Proof. See [19] (Theorem 7.1.4).

Theorem 7. Let $X$ be an injective Banach lattice with $\mathbb{B}:=\mathbb{M}(X)$ and let $\Phi$ be a strictly positive Maharam operator from $X$ to $\Lambda:=\Lambda(\mathbb{B})$ with the Levi property such that $\|x\|=\|\Phi(|x|)\|_{\infty}$ for all $x \in X$. Then

(1) For every $x^{\#} \in X^{\#}$ there is a unique orthomorphism $w \in \mathscr{Z}(X)$ such that $\left\langle\left\langle x, x^{\#}\right\rangle\right\rangle=$ $\Phi(w x)$ for all $x \in X$;

(2) $X^{\#}$ is a $\mathbb{B}$-AM-space and the mapping $x^{\#} \mapsto w$ is a $\mathbb{B}$-isometry from $X^{\#}$ onto $\mathscr{Z}(X)$;

(3) $\Phi^{\prime}$ is a lattice $\mathbb{B}$-isometry from $\Lambda^{\prime}$ onto a majorizing sublattice of $X^{\prime}$;

(4) $\left|x^{\prime}\right| \leq \Phi^{\prime}\left(\left|x^{\prime}\right|\right)$ For all $x^{\prime} \in X^{\prime}$; in particular, $x^{\prime}$ belongs to the $\sigma\left(X^{\prime}, X\right)$-closure of $\left\{\left|x^{\prime}\right| \circ \Phi \circ w: w \in \mathscr{Z}(X),|w| \leq I_{X}\right\}$.

Proof. If $x^{\#} \in X^{\#}$ then $\left|x^{\#}\right| \leq \lambda \Phi$ for some $\lambda \in \Lambda$. As $\lambda \Phi$ is a Maharam operator as well, there is $w^{\prime} \in \operatorname{Orth}(X)$ such that $\left|w^{\prime}\right| \leq I_{X}$ and $x^{\#}=\lambda \Phi w^{\prime}$ by Lemma 13. Considering that $\lambda \Phi=\Phi \lambda$ and putting $w=\lambda w^{\prime}$, we arrive at 7(1). The strict positivity of $\Phi$ implies the uniqueness of $w$ as well as the simultaneous positivity of $x^{\#}$ and $w$. Thus, the mapping $x^{\#} \mapsto w$ is a lattice isomorphism from $X^{\#}$ onto $\mathscr{Z}(X)$. By Lemma 14 we can identify $\Lambda$ with an order closed sub- $f$-algebra of $\mathscr{Z}(X)$ and define the $\Lambda$-valued norm on $\mathscr{Z}(X)$ as $|w|:=\inf \left\{\lambda \in \Lambda_{+}:|w| \leq \lambda\right\}$. Then the regular norm on $\mathscr{Z}(X)$ is a mixed norm: $\|w\|=\||w|\|_{\infty}$ for all $w \in \mathscr{Z}(X)$. It is clear from the above that $\left|x^{\#}\right| \leq \lambda$ if and only if $|w| \leq \lambda$ so that $\left|x^{\#}\right|=|w|$. Consequently, $\left\|\left|x^{\#}\||=|\| w\right|\right\|$, whence $7(2)$ holds.

The remaining part of the proof rests upon Lemma 15. Since $\Phi$ is interval preserving, $\Phi^{\prime}$ is a lattice homomorphism; see [16] (Theorem 2.19). Moreover, considering that $\Phi(X)=$ $\Lambda$ and $\Phi$ is $\Lambda$-linear, we deduce that

$$
\begin{gathered}
\left\|\Phi^{\prime} \lambda^{\prime}\right\|=\sup _{x \in X_{+},\|x\| \leq 1}\left\langle x, \Phi^{\prime} \lambda^{\prime}\right\rangle \\
=\sup _{x \in X_{+},\|\Phi(x)\|_{\infty} \leq 1}\left\langle\Phi(x), \lambda^{\prime}\right\rangle=\sup _{\lambda \in \Lambda_{+},\|\lambda\|_{\infty} \leq 1}\left\langle\lambda, \lambda^{\prime}\right\rangle=\|\lambda\|, \\
\pi^{\prime} \Phi^{\prime}=(\Phi \pi)^{\prime}=(\pi \Phi)^{\prime}=\Phi^{\prime} \pi^{\prime} .
\end{gathered}
$$

So, $\Phi^{\prime}$ is isometric and $\mathbb{B}$-linear. Now, 7(3) is immediate from the relations $\left\langle x, x^{\prime}\right\rangle \leq$ $\left\langle|x|,\left|x^{\prime}\right|\right\rangle=\left\langle\Phi(|x|),\left|x^{\prime}\right|\right\rangle=\left\langle|x|, \Phi^{\prime}\left|x^{\prime}\right|\right\rangle$ valid for all $x^{\prime} \in X^{\prime}$ and $x^{\prime} \in X^{\prime}$, according to which $\left|x^{\prime}\right| \leq \Phi^{\prime}\left(\left|x^{\prime}\right|\right)$. Define the continuous seminorm $p: X \rightarrow \mathbb{R}$ and the sublinear operator $P: X \rightarrow \Lambda$ as $p(x):=\left\langle\Phi(|x|),\left|x^{\prime}\right|\right\rangle$ and $P(x):=\Phi(|x|)$. By the Hahn-BanachKantorovich Theorem [16] (Theorem 1.25) for an arbitrary $x_{0} \in X$ there exists a linear operator $S: X \rightarrow \Lambda$ such that $S x_{0}=P\left(x_{0}\right)$ and $S x \leq P(x)$ for all $x \in X$. It follows that $S$ is regular, $|S| \leq \Phi$, and by Lemma 13 there exists an orthomorphism $w \in \mathscr{Z}(X)$ with $S x=\Phi(w x)$ for all $x \in X$ and $|w| \leq I_{X}$. Moreover, $P x_{0}=\Phi\left(w x_{0}\right)$ and we see that

$$
p(x)=\left\langle P(x),\left|x^{\prime}\right|\right\rangle=\sup \left\{\left\langle\Phi(w x),\left|x^{\prime}\right|\right\rangle: w \in \mathscr{Z}(X),|w| \leq I_{X}\right\} \quad(x \in X) .
$$

Finally, we conclude that $x^{\prime}$ lies in the dual closed unit ball of the seminorm $p$ and this unit ball is the $\sigma\left(X^{\prime}, X\right)$-closure of $\left\{\left|x^{\prime}\right| \circ \Phi \circ w: w \in \mathscr{Z}(X),|w| \leq I_{X}\right\}$.

We preface the next result with a few remarks. Denote by $\mathbb{P}_{L}^{*}\left(X^{\prime}\right)$ the part of $\mathbb{P}_{L}\left(X^{\prime}\right)$ consisting of $\sigma\left(X^{\prime}, X\right)$-continuous projections. Let $\mathscr{L}_{\mathbb{B}}^{*}\left(\Lambda^{\prime}, X^{\prime}\right)$ stand for the part of $\mathscr{L}\left(\Lambda^{\prime}, X^{\prime}\right)$ consisting of all continuous $\mathbb{B}$-linear operators from $\left(\Lambda^{\prime}, \sigma\left(\Lambda^{\prime}, \Lambda\right)\right)$ to $\left(X^{\prime}, \sigma\left(X^{\prime}, X\right)\right)$. Since $\Lambda^{\prime}$ is an $A L$-spaces and $X^{\prime}$ has the Levi property, every norm bounded linear operator from $\Lambda^{\prime}$ to $X^{\prime}$ is regular. Hence, $\mathscr{L}\left(\Lambda^{\prime}, X^{\prime}\right)$ coincides with $\mathscr{L}^{r}\left(\Lambda^{\prime}, X^{\prime}\right)$; see [34] (Theorem 3.5). Moreover, $\mathscr{L}^{r}\left(\Lambda^{\prime}, X^{\prime}\right)$ is a Dedekind complete Banach lattice. Now, every weak*-continuous linear operator from $\Lambda^{\prime}$ to $X^{\prime}$ is norm continuous and $\mathscr{L}_{\mathbb{B}}^{*}\left(\Lambda^{\prime}, X^{\prime}\right)$ is considered as a subspace of $\mathscr{L}^{r}\left(\Lambda^{\prime}, X^{\prime}\right)$ with the induced ordering. 
Theorem 8. Let $X$ be a Banach lattice, $\mathbb{B}$ a complete Boolean algebra, and $\Lambda=\Lambda(\mathbb{B})$. Then the following are equivalent:

(1) $X$ is injective with $\mathbb{B}:=\mathbb{P}_{M}(X)$.

(2) The four conditions hold:

(a) $\mathbb{B}$ is isomorphic to a complete subalgebra $\mathbb{P}_{L}^{*}\left(X^{\prime}\right)$ of the Boolean algebra $\mathbb{P}_{L}\left(X^{\prime}\right)$.

(b) There exists a lattice $\mathbb{B}$-isometry $J \in \mathscr{L}_{\mathbb{B}}^{*}\left(\Lambda^{\prime}, X^{\prime}\right)$ such that $J\left(\Lambda^{\prime}\right)$ is a majorizing sublattice of $X^{\prime}$.

(c) For every $x^{\prime} \in X^{\prime}$ there exists $\lambda^{\prime} \in \Lambda^{\prime}$ such that $\left|x^{\prime}\right| \leq J \lambda^{\prime},\left\|x^{\prime}\right\|=\left\|\lambda^{\prime}\right\|$, and $x^{\prime}$ lie in the $\sigma\left(X^{\prime}, X\right)$-closure of $\left\{S \lambda^{\prime}: S \in \mathscr{L}_{\mathbb{B}}^{*}\left(\Lambda^{\prime}, X^{\prime}\right),\|S\| \leq 1\right\}$.

(d) $\mathscr{L}_{\mathbb{B}}^{*}\left(\Lambda^{\prime}, X^{\prime}\right)$ is a $\mathbb{B}$-AM-space with $J$ as an order unit.

Proof. $(1) \Longrightarrow(2)$ : An injective Banach lattice $X$ is representable as $L^{1}(\Phi)$, where $\Phi: X \rightarrow$ $\Lambda$ is a strictly positive Maharam operator with the Levi property and $\mathbb{B}:=\mathbb{P}_{M}(X) \simeq \mathbb{P}(\Lambda)$; see 2.8. It is also clear that a band projection $\pi$ of $X$ lies in $\mathbb{P}_{M}(X)$ if and only if the adjoint $\pi^{\prime}$ lies in $\mathbb{P}_{L}\left(X^{\prime}\right)$. Moreover, $\pi^{\prime}$ is $\sigma\left(X^{\prime}, X\right)$-continuous. It follows that the mapping $\pi \mapsto \pi^{\prime}$ is a Boolean isomorphism from $\mathbb{P}(X)$ onto $\mathbb{P}_{L}^{*}\left(X^{\prime}\right)$. Put $J:=\Phi^{\prime}$ and observe that $J$ is a lattice isomorphism [16] (Theorem 2.20) and $J$ is an isometry:

$$
\begin{gathered}
\left\|J \lambda^{\prime}\right\|=\sup _{x \in X_{+},\|x\| \leq 1}\left\{\left\langle x, J\left|\lambda^{\prime}\right|\right\rangle\right\} \\
=\sup _{x \in X_{+}, \Phi(x) \leq \mathbb{1}}\left\{\left\langle\Phi(x),\left|\lambda^{\prime}\right|\right\rangle\right\}=\sup _{\lambda \in \Lambda_{+}, \lambda \leq \mathbb{1}}\left\{\left\langle\lambda,\left|\lambda^{\prime}\right|\right\rangle\right\}=\left\|\lambda^{\prime}\right\| .
\end{gathered}
$$

Thus, we have established $(a)$ and $(b)$, while $(c)$ and $(d)$ follow from Theorem 7 .

$(2) \Longrightarrow(1)$ : Assume that $(a)-(d)$ are fulfilled. From $(a)$ it follows that $\mathbb{P}_{M}(X)$ is isomorphic to $\mathbb{P}_{L}^{*}\left(X^{\prime}\right)$ as every projection in $\mathbb{P}_{L}^{*}\left(X^{\prime}\right)$ is of the form $\pi^{\prime}$ with $\pi \in \mathbb{P}_{M}(X)$ and the mapping $\pi \mapsto \pi^{\prime}$ is a norm preserving Boolean isomorphism from $\mathbb{P}_{M}(X)$ into $\mathbb{P}_{L}\left(X^{\prime}\right)$. Similarly, $\mathbb{P}_{M}(\Lambda)=\mathbb{P}(\Lambda)$ is Boolean isomorphic to $\mathbb{P}_{L}^{*}\left(\Lambda^{\prime}\right)$. Below it is convenient to identify all these five Boolean algebras.

According to $(b)$ there exists a positive operator $\Phi: X \rightarrow \Lambda$ with $J=\Phi^{\prime}$. Thus $\Phi$ is norm bounded and almost interval preserving by [18] (Theorem 1.4.19(ii)). Moreover, $\Phi(X)=\Lambda$ as $J$ is an isomorphism. Assuming that $\Phi(|x|)=0$ and using $(b)$, for an arbitrary $x^{\prime} \in X_{+}^{\prime}$ we may pick $\lambda^{\prime} \in \Lambda_{+}^{\prime}$ with $x^{\prime} \leq \Phi^{\prime} \lambda^{\prime}$ so that $0 \leq\left\langle|x|, x^{\prime}\right\rangle \leq\left\langle\Phi(|x|), \lambda^{\prime}\right\rangle=0$, whence $x=0$ and $\Phi$ is strictly positive. Thus we can define the $\Lambda$-valued norm on $X$ as follows: $|x|:=\Phi(|x|)$. Evidently $\|\Phi(|x|)\|_{\infty} \leq\|x\|$ as $\|\Phi\|=\|J\|=1$ and, as we will see later, the reverse inequality holds as well. Note that the norm $|\cdot|$ is decomposable; i.e., $\pi|x|=|\pi x|$ for all $x \in X$ and $\pi \in \mathbb{B}$ Indeed, for $x \in X, x^{\prime} \in X^{\prime}$, and $\pi \in \mathbb{B}$ we have $\left.\left\langle\pi \Phi(x), x^{\prime}\right\rangle=\left\langle x, J\left(\pi^{\prime} x^{\prime}\right)\right\rangle=\left\langle x, \pi^{\prime} J x^{\prime}\right\rangle=\left\langle\Phi(\pi x), x^{\prime}\right)\right\rangle$, whence $\pi \Phi(x)=\Phi(\pi x)$.

Using (c), we also define the $\Lambda^{\prime}$-valued norm on $X^{\prime}$ by putting $\left|x^{\prime}\right|=\lambda^{\prime}$ where $\left|x^{\prime}\right| \leq J \lambda^{\prime}$ and $\left\|x^{\prime}\right\|=\left\|\lambda^{\prime}\right\|$, so that $\left\|x^{\prime}\right\|=\left\|\left|x^{\prime}\right|\right\|$. The definition is sound, since this $\lambda^{\prime} \in \Lambda_{+}^{\prime}$ is unique. Indeed, if $\left|x^{\prime}\right| \leq J \lambda_{k}^{\prime}$ and $\left\|x^{\prime}\right\|=\left\|\lambda_{k}^{\prime}\right\|$ for $k=1$, 2; then $\left|x^{\prime}\right| \leq J \lambda_{1}^{\prime} \wedge$ $J \lambda_{2}^{\prime}=J\left(\lambda_{1}^{\prime} \wedge \lambda_{2}^{\prime}\right)$ and $\left\|\lambda_{k}^{\prime}\right\|=\left\|x^{\prime}\right\| \leq\left\|J\left(\lambda_{1}^{\prime} \wedge \lambda_{2}^{\prime}\right)\right\|=\left\|\lambda_{1}^{\prime} \wedge \lambda_{2}^{\prime}\right\| \leq\left\|\lambda_{k}^{\prime}\right\|$. It follows that $\left\|\lambda_{k}\right\|=\left\|\lambda_{1}^{\prime} \wedge \lambda_{2}^{\prime}\right\|$ and, since $\Lambda^{\prime}$ is an $A L$-space, $\left\|\lambda_{k}^{\prime}-\lambda_{1}^{\prime} \wedge \lambda_{2}^{\prime}\right\|=\left\|\lambda_{k}^{\prime}\right\|-\left\|\lambda_{1}^{\prime} \wedge \lambda_{2}^{\prime}\right\|=0$, so that $\lambda_{k}^{\prime}=\lambda_{1}^{\prime} \wedge \lambda_{2}^{\prime}$. Now, taking into account the inequality $\left|x^{\prime}\right| \leq J\left(\left|x^{\prime}\right|\right)$ we deduce

$$
\begin{aligned}
&\|x\|=\sup \left\{\left\langle x, x^{\prime}\right\rangle:\left\|x^{\prime}\right\| \leq 1\right\} \leq \sup \left\{\left\langle|x|, J\left|x^{\prime}\right|\right\rangle:\left\|x^{\prime}\right\| \leq 1\right\} \\
& \leq \sup \left\{\left\langle|x|, \lambda^{\prime}\right\rangle:\left\|\lambda^{\prime}\right\| \leq 1\right\}=\||x|\|_{\infty}
\end{aligned}
$$

and $\|x\|=\||x|\|_{\infty}$ for all $x \in X$ as claimed.

We will show that the Banach lattices $X^{\#}$ and $\mathscr{L}_{\mathbb{B}}^{*}\left(\Lambda^{\prime}, X^{\prime}\right)$ are lattice $\mathbb{B}$-isometric. Each $S \in \mathscr{L}_{\mathbb{B}}^{*}\left(\Lambda^{\prime}, X^{\prime}\right)$ is an adjoint operator, i.e., $S=T^{\prime}$ for some continuous operator $T \in$ $\mathscr{L}(X, \Lambda)$. Moreover, in this event for $C \in \mathbb{R}_{+}$we have $|T| \leq C \Phi$ if and only if $|S| \leq C J$. Thus, the mapping $T \mapsto T^{\prime}$ is an order preserving isometry from $X^{\#}$ onto $S \in \mathscr{L}_{\mathbb{B}}^{*}\left(\Lambda^{\prime}, X^{\prime}\right)$. This isometry is $\mathbb{B}$-linear, since $(\pi T)^{\prime}=T^{\prime} \pi=\pi T^{\prime}$, where $\pi$ denotes a band projection in 
$\Lambda$ as well as the corresponding projections in $\Lambda^{\prime}$ and $X^{\prime}$. It remains to refer to Theorem 6 to conclude that $X$ is an injective Banach lattice.

Remark 4. It should be emphasized that the sublattice $J\left(\Lambda^{\prime}\right)$ in $X^{\prime}$ admits a contractive positive projection. Indeed, there is an order continuous lattice isomorphism $\Psi: \Lambda \rightarrow X$ such that $\Phi \circ \Psi=I_{\Lambda}$; see [19] (Theorem 3.4.10). Therefore, $\Psi^{\prime} \circ \Phi^{\prime}=I_{\Lambda^{\prime}}$ and $P:=\Phi^{\prime} \circ \Psi^{\prime}$ is a contractive positive projection to $X^{\prime}$ with $P\left(X^{\prime}\right)=J\left(\Lambda^{\prime}\right)$.

Remark 5. In [7] (Proposition 3B) Haydon made the observation: If two hereditary downwards faces $G_{1}$ and $G_{2}$ of $U_{+}^{\prime}$, the positive part of the dual unit ball, have the properties (1) $G_{1}$ and $G_{2}$ are $\sigma\left(X^{\prime}, X\right)$-closed, (2) each $g \in U_{+}^{\prime}$ admits a unique representation $g=g_{1}+g_{2}$ with $g_{1} \in G_{1}$ and $g_{2} \in G_{2},(3)\left\|g_{1}+g_{2}\right\|=\left\|g_{1}\right\|+\left\|g_{2}\right\|$ for all $g_{1} \in G_{1}$ and $g_{2} \in G_{2}$; then there exists $\pi \in \mathbb{P}(X)$ such that $G_{1}=\pi^{\prime}\left(U_{+}^{\prime}\right)$ and $G_{2}=\left(I_{X}-\pi^{\prime}\right)\left(U_{+}^{\prime}\right)$. Of course in this case $\pi^{\prime} \in \mathbb{P}_{L}\left(X^{\prime}\right)$. Using this fact, item (a) of Theorem 8, can be formulated in terms of the facial structure of $U_{+}^{\prime}$.

\section{Concluding Remarks}

The geometric study of an $L^{1}$-predual space, a Banach space whose dual is isometric (isomorphic) to an $A L$-space, stems from the works of Grothendieck [35] and Lindenstrauss [36]. The integral representation theorems of Choquet led quite naturally to exploration of the related geometric structure of state spaces; see Alfsen [37]. One of the main problems in operator algebras is the geometric description of state spaces of operator algebras; the complete solution of this problem by Alfsen, Hanche-Olsen, Iohum, and Shultz is presented in the book [38]. One of the recent valuable developments is the classification of facially symmetric spaces which was initiated by Friedman and Rousso in [39]. In particular, the geometric description of an $L^{1}$-space as a strongly facially symmetric spaces given by Ibragimov and Kudaybergenov in [40] admits a counterpart for injective Banach lattices. This development of convexity theory has motivated the geometric study of the duals and preduals of injective Banach lattices.

In [41] (Corollary 1) Fremlin proved that a Banach lattice $X$ is isomorphic to an $L$-space if and only if every continuous linear operator from $X$ to $l^{1}$ is order bounded. To formulate the Boolean version of this result, we define the Boolean counterpart of the Banach lattice $l^{1}$. Suppose that $Q$ is the Stone representation space of $\mathbb{B}$ and $X$ is a Banach lattice. Denote by $C_{\#}(Q, X)$ the set of cosets of continuous vector-functions $u: \operatorname{dom}(u) \subset Q \rightarrow X$ such that $Q \backslash \operatorname{dom}(u)$ is a meager set in $Q$ and the continuous extension $|u|$ of the pointwise norm $q \mapsto\|u(q)\|$ to the whole of $Q$ belongs to the Banach lattice $C(Q)$ of continuous functions. Vector-functions $u$ and $v$ are equivalent if $u(q)=v(q)$ for all $q \in \operatorname{dom}(u) \cap \operatorname{dom}(v)$. If $\tilde{u}$ is the coset of $u$ then we define $|\tilde{u}|:=|u|$ and $\|\tilde{u}\|:=\||\tilde{u}|\|_{\infty}$. The set $C_{\#}(Q, X)$ is naturally endowed with the structure of a $\mathbb{B}$-complete Banach lattice; see 2.5. Moreover, $C_{\#}\left(Q, l^{1}\right)$ is an injective Banach lattice whose Boolean valued representation is $l^{1}$ within $\mathbb{V}^{(\mathbb{B})}$. Now, interpreting the Fremlin characterization of $L$-spaces in the model $\mathbb{V}^{(\mathbb{B})}$, we arrive at the following result.

Theorem 9. For a $\mathbb{B}$-cyclic Banach lattice $X$ the following are equivalent:

(1) There is a countable partition of unity $\left(\pi_{n}\right)_{n \in \mathbb{N}}$ in $\mathbb{B}$ such that $\pi_{n} X$ is lattice $\pi_{n} \mathbb{B}$ isomorphic to an injective Banach lattice for every $n \in \mathbb{N}$.

(2) Every continuous $\mathbb{B}$-linear operator from $X$ to $C_{\#}(Q, X)$ is order bounded.

If the dual $X^{\prime}$ of a Banach lattice $X$ is injective, then the pair $\left(X, X^{\prime}\right)$ with the conventional duality pairing admits a nice Boolean valued representation as a dual pair $\left(\mathscr{X}, \mathscr{X}^{\prime}\right)$ with $\mathscr{X}^{\prime}$ injective in an appropriate Boolean valued universe $\mathbb{V}^{(\mathbb{B})}$; see [11] (Theorem 3.8 and Corollary 3.9). Nevertheless, there is a striking asymmetry in these dualities: If a Banach lattice $X$ is injective, then the duality between $X$ and $X^{\prime}$ is not representable as Boolean valued duality between $\mathscr{X}$ and $\mathscr{X}^{\prime}$; see Theorem 6 . The reason is that for a dual space $X^{\prime}$ the Boolean algebra of $M$-projections is isomorphic to the complete Boolean 
algebra of $L$-projections on $X$, whilst the collection of $M$-projections on $X$ may be too scarce; see [42] (Theorems I.1.10 and V.4.6).

Theorem 7 can be viewed as a version for injective Banach lattices of Grothendieck's result stating that a Banach space is an $A L$-space provided that its dual is isomorphic to a Banach space of continuous functions on a locally compact space vanishing at infinity (see [43] (Theorem 27.4.1)); in other words, $L^{1}$ is the only predual of $L^{\infty}$ (see [35] ([Theorem 1)). As can be seen, the proof consists of a combination of Boolean valued representation and some results of the standard structure theory of Banach lattices. Another version of Grothendieck's result for injective Banach lattices in terms of the $\mathbb{B}$-dual $X^{\#}$ (Definition 7) can be obtained by the direct Boolean valued interpretation [9] (Theorem 5.12).

Author Contributions: Conceptualization, methodology, formal analysis, writing-original draft preparation, writing-review and editing by A.K. and S.K. All authors have read and agreed to the published version of the manuscript.

Funding: This research received no external funding.

Conflicts of Interest: The authors declare no conflict of interests.

\section{References}

1. Arendt, W. Factorization by lattice homomorphisms. Math. Z. 1984, 185, 567-571. [CrossRef]

2. Abramovich, Y.A. Injective envelopes of normed lattices. Dokl. Acad. Nauk SSSR 1971, 197, 743-745.

3. Lotz, H.P. Extensions and liftings of positive linear mappings on Banach lattices. Trans. Amer. Math. Soc. 1975, 211, 85-100. [CrossRef]

4. Lacey, H.E. The Isometric Theory of Classical Banach Spaces; Springer: Berlin/Heidelberg, Germany, 1974

5. Cartwright, D.I. Extension of positive operators between Banach lattices. Memoirs Amer. Math. Soc. 1975, 164, 1-48. [CrossRef]

6. Kusraev, A.G.; Kutateladze, S.S. Boolean Valued Analysis: Selected Topics. In Trends in Science: The South of Russia. A Math. Monogr. 6; SMI VSC RAS: Vladikavkaz, Russia, 2014.

7. Haydon, R. Injective Banach lattices. Math. Z. 1977 156, 19-47. [CrossRef]

8. Kusraev, A.G. Boolean valued analysis and injective Banach lattices. Dokl. Ross. Akad. Nauk 2012,444, 143-145. [CrossRef]

9. Kusraev, A.G. Boolean valued transfer principle for injective Banach lattices. Siberian Math. J. 2015, 25, 57-65. [CrossRef]

10. Kusraev, A.G. Injective Banach lattices: A survey. Eurasian Math. J. 2014, 5, 58-79.

11. Kusraev, A.G.; Kutateladze, S.S. Geometric characterization of preduals of injective Banach lattices. Indag. Math. 2020, 31, 863-878. [CrossRef]

12. Buskes, G. Separably injective Banach lattices are injective. Proc. Roy. Irish Acad. Sect. A 1985, 85, 185-186.

13. Lindenstrauss, J.; Tzafriri, L. On the isomorphic classification of injective Banach lattices. In Mathematical Analysis and Applications, Part B; Nachbin, L., Ed.; Acad. Press: New York, NY, USA; London, UK, 1981; pp. 489-498, (Adv. in Math. Suppl. Stud., 7b).

14. Mangheni, P.J. The classification of injective Banach lattices. Israel J. Math. 1984, 48, 341-347. [CrossRef]

15. Wickstead, A.W. Relatively central operators on Archimedean vector lattices. Proc. Roy. Irish Acad. Sect. A 1980, 80, 191-208.

16. Aliprantis, C.D.; Burkinshaw, O. Positive Operators; Academic Press Inc.: London, UK, 1985.

17. Luxemburg, W.A.J.; Zaanen, A.C. Riesz Spaces. 1; North Holland: London, UK, 1971.

18. Meyer-Nieberg, P. Banach Lattices; Springer: Berlin/Heidelberg, Germany, 1991.

19. Kusraev, A.G. Dominated Operators; Kluwer: Dordrecht, The Netherlands, 2000.

20. Bell, J.L. Boolean-Valued Models and Independence Proofs in Set Theory; Clarendon Press: New York, NY, USA, 1985.

21. Kusraev, A.G.; Kutateladze, S.S. Boolean Valued Analysis; Kluwer: Dordrecht, The Netherlands, 1999.

22. Takeuti, G.; Zaring, W.M. Axiomatic Set Theory; Springer: New York, NY, USA, 1973.

23. Gordon, E.I. Real numbers in Boolean-valued models of set theory and K-spaces. Dokl. Akad. Nauk SSSR. 1977, 237, 773-775.

24. Takeuti, G. Boolean Valued Analysis; Springer: Berlin/Heidelberg, Germany, 1979; pp. 714-731.

25. Takeuti, G. Two Applications of Logic to Mathematics; Princeton University Press: Princeton, NJ, USA, 1978.

26. Abramovich, Y.A.; Aliprantis, C.D. Positive operators. In Handbook of the Geometry of Banach Spaces, 1; Johnson, W.B., Lindenstrauss, J., Eds.; Elsevier Science B.V.: Amsterdam, The Netherlands, 2001; pp. 85-122.

27. Ozawa, M. Boolean valued interpretation of Banach space theory and module structures of von Neumann algebras. Nagoya Math. J. 1990, 117, 1-36. [CrossRef]

28. Wong, Y.-C.; Ng, K.-F. Partially Ordered Topological Vector Spaces; Clarendon Press: Hong Kong, China, 1973.

29. Asimow, L.; Ellis, A.J. Convexity Theory and Its Applications in Functional Analysis. In London Mathematical Society Monograph 16; Academic Press: London, UK, 1980

30. Saito, K.; Wright, J.D.M. Monotone Complete C*-algebras and Generic Dynamics; Springer: London, UK, 2015.

31. Timofte, V. An isomorphic characterization of $L^{1}$-spaces. Indag. Mathem. 2007, 18, 629-640. [CrossRef]

32. Schaefer, H.H. Banach Lattices and Positive Operators; Springer: Berlin/Heidelberg, Germany, 1974. 
33. Luxemburg, W.A.J.; Schep, A. A Radon-Nikodým type theorem for positive operators and a dual. Indag. Math. 1978, 40, 357-375. [CrossRef]

34. Abramovich, Y.A.; Wickstead, A.W. When each continuous operator is regular, II. Indag. Math. N.S. 1997, 8, 281-294. [CrossRef]

35. Grothendieck, A. Une caractérisation vectorielle-métrique des espaces $L^{1}$. Canadian J. Math. 1955, 7, 552-561. [CrossRef]

36. Lindenstrauss, J. Extensions of compact operators. Memoirs Amer. Math. Soc. 1964, 48, 1-112. [CrossRef]

37. Alfsen, E.M. Compact Convex Sets and Boundary Integrals; Springer: Berlin/Heidelberg, Germany, 1971.

38. Alfsen, E.M.; Shultz, F.W. Geometry of State Spaces of Operator Algebras; Springer: New York, NY, USA, 2003.

39. Friedman, Y.; Russo, B. Geometry of the Dual Ball of the Spin Factor. Proc. Lond. Math. Soc. 1992, 65, 142-174. [CrossRef]

40. Ibragimov, M.M.; Kudaibergenov, K.K. Geometric description of $L^{1}$-spaces. Russian Math. (Iz. VUZ) 2013, 57, 16-21. [CrossRef]

41. Fremlin, D.H. A characterization of L-spaces. Indag. Math. 1974, 77, 270-275. [CrossRef]

42. Harmand, P.; Werner, D.; Wener, W. M-Ideals in Banach Spaces and Banach Algebras; Springer: Berlin/Heidelberg, Germany, 1993; (Lecture Notes in Math, 1547).

43. Semadeni, Z. Banach Spaces of Continuous Functions; Polish Scientific Publishers: Warszawa, Poland, 1971 ; Volume 1. 